\title{
MAXIMAL POSITIVE BOUNDARY VALUE PROBLEMS AS LIMITS OF SINGULAR PERTURBATION PROBLEMS ${ }^{1}$ \\ BY
}

\author{
CLAUDE BARDOS AND JEFFREY RAUCH
}

\begin{abstract}
We study three types of singular perturbations of a symmetric positive system of partial differential equations on a domain $\Omega \subset \mathbf{R}^{n}$. In all cases the limiting behavior is given by the solution of a maximal positive boundary value problem in the sense of Friedrichs. The perturbation is either a second order elliptic term or a term large on the complement of $\Omega$. The first corresponds to a sort of viscosity and the second to physical systems with vastly different properties in $\Omega$ and outside $\Omega$. The results show that in the limit of zero viscosity or infinitely large difference the behavior is described by a maximal positive boundary value problem in $\Omega$. The boundary condition is determined in a simple way from the system and the singular terms.
\end{abstract}

1. Introduction. In this paper we study boundary value roblems for singularly perturbed systems of partial differential equations. The unperturbed system is of first order and positive symmetric in the sense of Friedrichs. We study three sorts of singular perturbations: (1) the addition of $\varepsilon$ times a positive second order elliptic system in a domain $\Omega,(2,3)$ the addition of $\lambda P$ or $\lambda P(\partial / \partial t)$ with $\lambda \gg 1$ and $P$ strictly positive in the exterior of $\Omega$ and zero in $\Omega$. In the first case we study the solutions $u_{\varepsilon}$ to the Dirichlet problem in $\Omega$. Bardos, Brezis and Brezis [1] have shown that in this case $u_{\varepsilon}-u$ weakly in $L^{2}(\Omega)$ where $u$ is uniquely determined as the solution of a maximal positive boundary value problem for the unperturbed operator. Here one has the phenomenon of loss of boundary conditions in the limit and the existence of boundary layers for $u_{\varepsilon}$ near $\partial \Omega$. We prove several more refined estimates on $u_{\varepsilon}$, in particular a uniform bound in $H^{1 / 2-\eta}(\Omega)$ for any $\eta>0$.

These estimates yield a proof of the strong convergence of $u_{\varepsilon}$ to $u$ in all $H^{s}(\Omega)$ for $s<\frac{1}{2}$. This singular perturbation problem is well known and much studied, in contrast to the problems of the second sort mentioned above. In the latter problems we study the Cauchy problems

$$
\begin{aligned}
(L+\text { perturbation }) u_{\lambda} & =0 \text { on }[0, T] \times \mathbf{R}^{n}, \\
u(0, \cdot) & =g(x) \text { on } \mathbf{R}^{n},
\end{aligned}
$$

Received by the editors July 27, 1978 and, in revised form, August 8, 1980.

1980 Mathematics Subject Classification. Primary 35B25, Secondary 35F15, 35L50.

Key words and phrases. Singular perturbations, boundary layers, maximal positive boundary value problems.

${ }^{1}$ Research supported by the National Science Foundation under grant number NSF MCS 79-01857. 
describing wave propagation on all of $\mathbf{R}^{n}$. The perturbation is very large on the complement of a region $[0, T] \times \Omega$. The phenomenon we find is that as $\lambda \rightarrow \infty$,

$$
\begin{array}{ll}
u_{\lambda} \rightarrow 0 & \text { outside }[0, T] \times \Omega, \\
u_{\lambda} \rightarrow u & \text { in }[0, T] \times \Omega,
\end{array}
$$

where $u$ is uniquely determined as the solution of a maximal positive boundary value problem on $[0, T] \times \Omega$ with homogeneous boundary conditions on $[0, T] \times \partial \Omega$ determined by the operator $L$ and the matrix $P$ on $[0, T] \times \partial \Omega$. Note that in the first problems we passed from one boundary value problem $\Omega$ to a limiting boundary value problem on $\Omega$ while in the present context one passes from a Cauchy problem on $[0, T] \times \mathbf{R}^{n}$ to a mixed initial boundary value problem on $[0, T] \times \Omega$.

In problems of wave propagation one often introduces mixed problems on a domain $[0, T] \times \Omega$ where the conditions on $[0, T] \times \partial \Omega$ describe the interaction of waves with the boundary. Invariably such models involve idealizations about the containing walls $[0, T] \times \partial \Omega$. In reality waves always penetrate beyond the walls. $\mathrm{A}$ more complete model would involve an equation on $[0, T] \times \mathbf{R}^{n}$ which suffers an abrupt change at $[0, T] \times \partial \Omega$ reflecting the fact that the medium in $\Omega$ is very different from that in the walls. What is needed then is a theorem asserting that in $[0, T] \times \Omega$ the solution is approximated by solutions of the mixed problem which one has proposed as a model. Our theorems provide rigorous results of this type. This point of view is presented in an unpublished set of lecture notes by Friedrichs [4] where he predicts, by a formal asymptotic analysis, the results we prove.

Notice that for all three singular perturbation problems considered above the limiting problem is a maximal positive boundary value problem in the sense of Friedrichs [3]. This has two important consequences. First, the limiting boundary value problems are well posed. This is a crucial fact, since in reality one never observes the limit $\lambda \rightarrow \infty$ or $\varepsilon \rightarrow 0$, only $\lambda$ very large or $\varepsilon$ very small. Then the quantities inside the region do not satisfy the homogeneous boundary condition $M u=0$ but do have $M u$ small. Since the boundary value problem is well set, it follows that $u$ differs from the limiting solutions by an amount which can be estimated in terms of $M u$. Thus if one observes that there is very little disturbance outside $\Omega$ this is sufficient to justify the use of the limiting equations. From a practical point of view, this gives a criterion for knowing when to use the limiting equations. Second, a special place is assumed by the maximal positive boundary conditions among the many well-set boundary value problems. Since "physical" boundary conditions arise as limits when some parameter is large, we expect the basic boundary value problems of mathematical physics to be maximal positive.

It should be remarked that, corresponding to the fact that our perturbations are strictly positive, the boundary conditions we find are not only positive but strictly positive. If the boundary matrix $A_{\nu}$ is nonsingular this means $\left\langle A_{\nu} u, u\right\rangle \geqslant c|u|^{2}$ for all vectors $u$ in the appropriate boundary space. In particular one does not find conservative boundary conditions, which satisfy $\left\langle A_{\nu} u, u\right\rangle=0$, with these methods.

There are a few well-understood problems which lead to conservative conditions: Dirichlet + Neumann problems for $u_{t t}-\Delta u$ and some problems in elasticity [4]. 
For these problems the positive definiteness of the perturbation is relaxed to positive semidefiniteness, and at present we do not have a good general theory encompassing these examples. On the other hand the inverse problem has a successful resolution in the category of strictly positive boundary conditions. That is, given a strictly positive boundary condition, there exist singular perturbations of all three kinds which yield this boundary condition in the limit. The interested reader is referred to [17] for the proof.

Some of the problems we discuss have discontinuous coefficients, large in one region and not large in an adjacent region. One can argue that this too is an idealization and that one should replace such discontinuities by narrow regions where the coefficients change rapidly. Our methods can be adapted to treat such problems provided that in the appropriate limit the width of the narrow regions shrinks to zero rapidly. The details of this modification will not be described.

An important ingredient in the proof of all of the theorems are estimates, uniform in the perturbation parameter, of the derivatives of solutions in directions parallel to the boundaries. This is consistent with the emergence of a boundary layer where quantities change rapidly as one moves away from the boundary and slowly as one moves parallel to the boundary (see $[\mathbf{1 4}, \mathbf{1 5}]$ for a discussion of boundary layers for problems analogous to ours).

The paper is organized as follows. In $\$ 2$ we give the notations and we state the theorems. Theorem 1 is concerned with the limit of vanishing viscosity. Theorems 2-4 deal with the results predicted by Friedrichs. In $\$ 3$ we prove weak convergence for Theorems $2-4$. $\$ 4$ is devoted to the tangential regularity theorems whose proofs have a common thread. In all cases the idea is to take the given equation, differentiate tangentially and then apply the energy method. The crux is that by an appropriate change of independent and dependent variables one can cast the given differential operator into a special form so that the commutator with tangential differentiation is suitably small. Given these results the proof of strong convergence is not difficult, with the exception of the problem of vanishing viscosity. Here the proof of the $H^{1 / 2-\eta}$ estimate requires new ideas and is especially troublesome when $\partial \Omega$ is characteristic for the unperturbed system. The proofs of strong convergence and the $H^{1 / 2-\eta}$ estimates are located in $\S 5$.

ACKNOWLEDGMENT. In our research we have benefitted from the careful reading and thoughtful criticisms of $J$. Ralston. We offer him our hearty thanks.

2. Notations and statements of the results. Let $L$ be the symmetric system of partial differential operators

$$
L=\sum_{1}^{n} A_{j}(x) \frac{\partial}{\partial x_{j}}+B(x) .
$$

That is, $A_{j} \in C^{3}\left(\mathbf{R}^{n}\right)$ and $B \in C^{2}\left(\mathbf{R}^{n}\right)$ are $k \times k$ matrix-valued functions with $A_{j}(x)$ hermitian symmetric for all $x \in R^{n}$. We assume that $D^{\beta} B$ and $D^{\alpha} A_{j}$ for $j=1, \ldots, n$, $|\alpha| \leqslant 3,|\beta| \leqslant 2$, are uniformly bounded on $\mathbf{R}^{n}$. We denote by

$$
\delta_{0} \equiv \frac{1}{2} \inf _{x \in \mathbf{R}^{n}} \min \left\{\lambda: \lambda \in \operatorname{spectrum} B(x)+B^{*}(x)-\sum_{j} \frac{\partial A_{j}(x)}{\partial x_{j}}\right\} .
$$


$\Omega$ will denote an open set of $\mathbf{R}^{n}$ which lies on one side of its smooth boundary $\partial \Omega$. We do not assume that $\Omega$ is bounded but we do assume that $\partial \Omega$ is bounded.

A smooth boundary space $N$ is a continuously differentiable map defined in a neighborhood of $\partial \Omega$ with value in $\Gamma_{p}\left(C^{k}\right)$, the manifold of $p$-dimensional linear subspaces of $\mathbf{C}^{k}$.

For any $m \times m$ hermitian matrix $B$ we denote by $B^{0}$ the kernel of $B$, by $B^{ \pm}$the space spanned by the eigenvectors with strictly positive (negative) eigenvalues $\left(\mathbf{C}^{m}=B^{+} \oplus B^{0} \oplus B^{-}\right)$. We denote by $M(B)$ the space $B^{+} \oplus B^{0}$.

We assume that there is a $C^{3}$ vector field $\nu(x)$ defined in a neighborhood of $\partial \Omega$ with the following properties: (1) on $\partial \Omega, \nu(x)$ coincides with the unit normal to $\partial \Omega$ which points out of $\bar{\Omega} ;(2)$ on a neighborhood of each connected component of $\partial \Omega$ there are matrix-valued functions $U, a_{+}, a_{-}$, of class $C^{3}$ with $U$ unitary and $\pm a_{ \pm}$ strictly positive and diagonal so that

$$
A_{\nu}(x) \stackrel{\text { def }}{=} \sum \nu_{j}(x) A_{j}(x)=U^{*}(x)\left(\begin{array}{ccc}
a_{+}(x) & 0 & 0 \\
0 & a_{-}(x) & 0 \\
0 & 0 & 0
\end{array}\right) U(x) .
$$

In particular, the dimensions of $A_{\nu}^{0}, A_{\nu}^{+}, A_{\nu}^{-}$and $M\left(A_{\nu}\right)$ are constant on each such neighborhood.

Concerning norms and inner products, \|\|$_{X}$ will denote the norm in the space $X$, $\|$ and $\langle$,$\rangle the norm and inner product in \mathbf{C}^{k}$ and $($,$) or (,)_{2}$ the inner product in $L^{2}(\mathscr{U})$. We use the symbol $L^{2}(\mathcal{Q})$ for both scalar and $\mathbf{C}^{k}$-valued $L^{2}$ spaces. Thus

$$
(f, f)_{\mathcal{Q}}=\int_{\mathcal{Q}}\langle f, f\rangle d x .
$$

A subspace $N$ of $\mathbf{C}^{k}$ is maximal positive for a matrix $B$ if and only if

$$
\begin{gathered}
\langle B \eta, \eta\rangle \geqslant 0 \quad \forall \eta \in N, \\
\operatorname{dim} N=\operatorname{dim} M(B) .
\end{gathered}
$$

The classical result is the following: Let $N(x)$ be a smooth boundary space; assume that $N(x)$ is for any $x \in \partial \Omega$ a maximal positive subspace for $A_{v}$, then (see $[3,8,11]$ ) for every $f \in L^{2}(\Omega)$ and $\delta>\delta_{0}$, there exists a unique $u$ which is a solution of the following problem:

$$
\delta u+L u=f \quad \text { in } \Omega,\left.\quad u\right|_{\partial \Omega} \in N .
$$

When (2.3) is satisfied, it is clear that $M\left(A_{\nu}\right)$ is a maximal positive smooth boundary space. Under the same hypothesis it is also true (see [1]) that if $E(x)$ is a smooth positive hermitian matrix,

$$
E^{-1 / 2}(x)\left(M\left(E^{-1 / 2}(x) A_{\nu}(x) E^{-1 / 2}(x)\right)\right)
$$

is a maximal positive subspace and therefore there exists for every $f \in L^{2}(\Omega)$ a unique solution $u \in L^{2}(\Omega)$ of the following problem:

$$
\delta u+L u=f,\left.\quad E^{1 / 2} u\right|_{\partial \Omega} \in M\left(E^{-1 / 2} A_{\nu} E^{-1 / 2}\right) .
$$

Finally we recall some facts about traces (see [1]). If for each $x \in \partial \Omega$ we write $\mathbf{C}^{k}=\operatorname{Ker}\left(A_{\nu}\right) \oplus\left(\operatorname{Ker} A_{\nu}\right)^{\perp}$, then for any $u \in L_{2}(\Omega)$ with $L u \in L_{2}(\Omega)$ the projection of $\left.u\right|_{\partial \Omega}$ on $\left(\operatorname{Ker} A_{\nu}\right)^{\perp}$ is well defined as an element of $H_{-1 / 2}(\partial \Omega)$. Since 
$\operatorname{Ker} A_{\nu} \subset N$ for any maximal positive subspace $N$, if $L u \in L_{2}(\Omega)^{k}$ the condition $\left.u\right|_{\partial \Omega} \in N$ can be made precise in two equivalent ways. First using traces we may compute the projection of $\left.u\right|_{\partial \Omega}$ onto $N^{\perp}$ and insist that it vanish, and second, we could demand that the relation

$$
\int_{\Omega}\langle v, L u\rangle d x=\int_{\Omega}\left\langle L^{*} v, u\right\rangle d x
$$

hold for all $v \in C_{(0)}^{1}(\bar{\Omega})$ such that $A_{\nu}(x) v(x) \in N(x)^{\perp}$ for all $x \in \partial \Omega$. Here $L^{*}$ is the formal adjoint differential operator to $L$.

Now we give the main statements.

THEOREM 1. Assume that $\delta>\delta_{0}$ and that $E_{i j}(x)$ is a family of $n^{2}$ hermitian matrices with the following properties:

(i) The matrices $D^{\alpha} E_{i j},|\alpha| \leqslant 2$, are bounded and continuous on a neighborhood of $\bar{\Omega}$.

(ii) For every unit vector $\xi=\left(\xi_{1}, \xi_{2}, \ldots, \xi_{n}\right) \in \mathbf{R}^{n}$ the matrix $E \xi \cdot \xi \stackrel{\text { def }}{=} \Sigma_{i j} E_{i j} \xi_{i} \xi_{j}$ is uniformly strictly positive in $\Omega$.

Then, for every $f \in L^{2}(\Omega)$, the sequence $u_{\varepsilon}$ of solutions to

$$
-\varepsilon \sum_{i j} \frac{\partial}{\partial x_{i}} E_{i j} \cdot \frac{\partial u_{\varepsilon}}{\partial x_{j}}+\delta u_{\varepsilon}+L u_{\varepsilon}=f,\left.\quad u_{\varepsilon}\right|_{\partial \Omega}=0,
$$

converges strongly in $L^{2}(\Omega)$, when $\varepsilon$ goes to zero to the solution of the following problem:

$$
\delta u+L u=f,\left.\quad(E \nu \cdot \nu)^{1 / 2} u\right|_{\partial \Omega} \in M\left((E \nu \cdot \nu)^{-1 / 2} A_{\nu}(E \nu \cdot \nu)^{-1 / 2}\right) .
$$

THEOREM 2. Let $\delta>\delta_{0}$ and $P$ a twice continuously differentiable hermitian symmetric-valued function on the complement, $\Omega^{c}$, of $\Omega$ and suppose all first order partial derivatives of $P$ are bounded on $\Omega^{c}$. We suppose that $P$ is uniformly strictly positive on $\Omega^{c}$. We denote also by $P$ the function obtained by setting $P(x)=0$ for $x \in \Omega$; then for every $f \in L^{2}\left(\mathbf{R}^{n}\right)$, the solution $u_{\lambda}$ of the problem

$$
\delta u_{\lambda}+L u_{\lambda}+\lambda P u_{\lambda}=f \text { in } \mathbf{R}^{n}
$$

converges strongly in $L^{2}\left(R^{n}\right)$, when $\lambda$ goes to infinity, to the limit $u$ defined by the relations:

$$
\begin{aligned}
& u=0 \text { in } \Omega^{c}, \quad u=\tilde{u} \quad \text { in } \Omega \\
& \delta \tilde{u}+L \tilde{u}=f \quad \text { in } \Omega,\left.\quad P^{1 / 2} \tilde{u}\right|_{\partial \Omega} \in M\left(P^{-1 / 2}\left(A_{\nu}\right) P^{-1 / 2}\right) .
\end{aligned}
$$

Henceforth we will assume that the operator $L$ depends also on the parameter $t \in[0, T]$ and we will write

$$
L(t)=A_{0}(t, x) \frac{\partial}{\partial t}+\sum_{1}^{n} A_{j}(t, x) \frac{\partial}{\partial x_{j}}+B(t, x)
$$

where $A_{j}$ and $B$ are $k \times k$ matrix-valued functions on $[0, T] \times \mathbf{R}^{n}$ with $A_{j}$ hermitian symmetric and $D_{t, x}^{\alpha} A_{j}, D_{t, x}^{\beta} B$ bounded and continuous on $[0, T] \times \mathbf{R}^{n}$ for $|\alpha| \leqslant 3$, $|\beta| \leqslant 2,0 \leqslant j \leqslant n$. The critical hyperbolicity assumption is

$$
A_{0}(t, x) \geqslant \delta_{1} I>0 \quad \forall(t, x) \in[0, T] \times \mathbf{R}^{n} .
$$


We consider two problems involving a large parameter $\lambda$ :

$$
(L+\lambda P(t, x)) u_{\lambda}=f \text { in }[0, T] \times \mathbf{R}^{n}
$$

and

$$
\left[L+\lambda \tilde{A}_{0}(t, x)(\partial / \partial t)\right] u_{\lambda}=f \quad \text { in }[0, T] \times \mathbf{R}^{n}
$$

with Cauchy data

$$
u_{\lambda}(0, \cdot)=g(\cdot) \quad \text { in }\{0\} \times \mathbf{R}^{n}, \quad \operatorname{supp} g(\cdot) \subset \bar{\Omega}
$$

where $\tilde{A}_{0}$ and $P$ are uniformly positive matrices on $\Omega^{c}$ and vanish in $\Omega$. In either case, $u_{\lambda}$ is uniquely determined and $u_{\lambda} \rightarrow 0$ on $\Omega^{c}$. The problem is to characterize $\lim u_{\lambda}=u$ as the solution of a boundary value problem on $[0, T] \times \Omega$. We have

THEOREM 3. Assume that the matrix-valued function $P$ has the following properties:

$$
\left\{\begin{array}{l}
P=0 \text { on } \Omega \text { and there is } a \gamma>0 \\
\quad \text { such that } P(t, x) \geqslant \gamma I \quad \forall(t, x) \in[0, T] \times \Omega^{c} . \\
P \in C^{1}\left([0, T] \times \bar{\Omega}^{c}\right) \text { and for }|\alpha|=1, D_{t, x}^{\alpha} P \\
\quad \text { is bounded on }[0, T] \times \bar{\Omega}^{c} .
\end{array}\right.
$$

Assume that $f \in L^{2}\left([0, T] \times \mathbf{R}^{n}\right), g \in L^{2}\left(\mathbf{R}^{n}\right)$. Denote by $u_{\lambda}$ the solution of the Cauchy problem (2.12), (2.14) by $\bar{u}$ the solution of the problem

$$
\left\{\begin{array}{l}
L \bar{u}=f \quad \text { in }[0, T] \times \Omega, \quad \bar{u}(0, \cdot)=g(\cdot) \quad \text { in } \Omega, \\
P^{1 / 2} \bar{u} \in M\left(P^{-1 / 2} A_{\nu} P^{-1 / 2}\right) \quad \text { in }[0, T] \times \partial \Omega,
\end{array}\right.
$$

and by $u$ the function

$$
u= \begin{cases}0 & \text { if } x \in \Omega^{c}, \\ \bar{u}(x) & \text { if } x \in \Omega .\end{cases}
$$

Then as $\lambda \rightarrow \infty, u_{\lambda} \rightarrow u$ in $C\left([0, T] ; L^{2}\left(\mathbf{R}^{n}\right)\right)$.

To study the limit in problem (2.13) we will have to impose positivity and regularity hypotheses analogous to (2.15). Precisely we assume

Positivity. $\tilde{A}_{0}(t, x)=0$ if $x \in \Omega$ and there is a $\gamma>0$ such that $\tilde{A}_{0}(t, x) \geqslant \gamma I$ if $x \in \Omega^{c}$.

Regularity. For $|\alpha| \leqslant 2, D_{t, x}^{\alpha} \tilde{A}_{0}$ is uniformly bounded on $[0, T] \times \Omega^{c}$.

However, reflecting the fact that the perturbation in (2.13) is more singular than in the other problems, we will also have to restrict the manner in which $\tilde{A}_{0}$ and $A_{\nu}$ vary with time. The result we obtain is false without some such restriction, as we show by example in Remark 6 following the statement.

Restricted dependence on time. For all $(t, x)$ with $x \in \partial \Omega$ the matrix $\left(\tilde{A}_{0}\right)^{1 / 2}\left(\tilde{A}_{0}^{-1 / 2}\right)_{t}$ maps the eigenspace $M\left(\tilde{A}_{0}^{-1 / 2} A_{\nu} \tilde{A}_{0}^{-1 / 2}\right)$ into itself. Let $K_{-}$be orthogonal projection onto the complement of this space. For $x \in \partial \Omega, \partial K_{-} / \partial t$ also maps $M\left(\tilde{A}_{0}^{-1 / 2} A_{\nu} \tilde{A}_{0}^{-1 / 2}\right)$ into itself. 
This condition is surely satisfied if $\tilde{A}_{0}$ and $\tilde{A}_{\nu}$ do not depend on time. More generally if there are matrix-valued functions $R_{0}(x)$ and $R_{1}(x)$ on $\partial \Omega$, scalar valued functions $c_{0}, c_{1}$ on $[0, T] \times \partial \Omega$ all smooth with

$$
\begin{aligned}
& \tilde{A}_{0} \uparrow[0, T] \times \partial \Omega=c_{0}(t, x) R_{0}(x), \\
& A_{\nu} \uparrow[0, T] \times \partial \Omega=c_{1}(t, x) R_{1}(x),
\end{aligned}
$$

Then on $\partial \Omega,\left(\tilde{A}_{0}\right)^{1 / 2}\left(\tilde{A}_{0}^{-1 / 2}\right)_{t}=c_{0}^{1 / 2}\left(\partial c_{0}^{-1 / 2} / \partial t\right) I$ and $K_{-}$is independent of $t$. This example gives the spirit of the restriction; it is a restriction on the amount of twisting.

THEOREM 4. Assume that $\tilde{A}_{0}$ satisfies the positivity, regularity and restricted dependence on time hypotheses above. Assume that $f \in L^{2}\left([0, T] \times \mathbf{R}^{n}\right)$ and $g \in L^{2}\left(\mathbf{R}^{n}\right)$, $\left.g\right|_{\Omega^{c}}=0$. Denote by $u_{\lambda}$ the solution of the Cauchy problem (2.13), (2.14), ${ }^{2}$ and by $\bar{u}$ the solution of the problem

$$
\left\{\begin{array}{l}
L \bar{u}=f \quad \text { in }[0, T] \times \Omega, \quad \bar{u}(0, \cdot)=g(\cdot) \quad \text { in } \Omega, \\
\tilde{A}_{0}^{1 / 2} \bar{u} \in M\left(\tilde{A}_{0}^{-1 / 2} A_{\nu} \tilde{A}_{0}^{-1 / 2}\right) \quad \text { in }[0, T] \times \partial \Omega .
\end{array}\right.
$$

Then as $\lambda \rightarrow \infty, u_{\lambda} \rightarrow u$ in $C\left([0, T] ; L^{2}\left(\mathbf{R}^{n}\right)\right)$, where $u$ is defined by the relation

$$
u=0 \quad \text { if } x \in \Omega^{c}, \quad u=\bar{u} \quad \text { if } x \in \Omega .
$$

REMARKS. 1. Theorem 1 has been considered in [1] but only the weak convergence is proved there. Strong convergence and the $H^{1 / 2-\eta}$ estimates were proved by Kato [7] in less generality (assuming that the matrix $\nu \cdot A$ is nonsingular on the boundary).

2. Theorems 2 and 4 provide proofs for two results predicted by Friedrichs in [4].

3. In the case $A_{0}, A_{j}, B$ and $P$ do not depend on $t$. Theorem 3 follows from Theorem 2 by a variant of the Trotter-Kato convergence theorem (see [10, p. 52] for a similar argument). In the same vein one could consider the parabolic system

$$
\begin{gathered}
\frac{\partial u_{\varepsilon}}{\partial t}-\varepsilon \sum \frac{\partial}{\partial x_{i}} E_{i j} \frac{\partial u_{\varepsilon}}{\partial x_{j}}+\delta u_{\varepsilon}+L u_{\varepsilon}=f \quad \text { in }[0, T] \times \Omega, \\
\left.u_{\varepsilon}\right|_{[0, T] \times \partial \Omega}=0, \quad u_{\varepsilon}(0, \cdot)=g(\cdot)
\end{gathered}
$$

as $\varepsilon \rightarrow 0$. If the coefficients are independent of $t$, the Trotter-Kato theorem together with Theorem 1 describes the limit. If the coefficients depend on $t$, a separate argument is required. This problem will not be discussed though no essentially different ideas are required.

4. The problems discussed in Theorem 4 pose some interesting technical difficulties. For example, because of the discontinuity of $A_{0}+\lambda \tilde{A}_{0}$ on $\partial \Omega$, the term $\left(A_{0}+\lambda \tilde{A}_{0}\right) \partial u / \partial t$ is not a well-defined distribution for $u \in L^{2}\left([0, T] \times \mathbf{R}^{n}\right)$. Since the large coefficient is in the terms of highest order, this perturbation is, in a sense, more singular than the others. In addition, it is interesting to observe the fundamental importance of the coefficient of $\partial / \partial t$ in the physical applications. It not only defines the energy but in many problems is the only coefficient which depends on $x$

\footnotetext{
${ }^{2}$ The existence and uniqueness of the solution of $(2.13),(2.14)$ are discussed in $\$ 3$.
} 
and $t$. The latter point is emphasized in Wilcox [16] where one can find many examples. Using these as a starting point a variety of examples illustrating Theorems $1-4$ are easily constructed.

5. The physical mechanism excluding waves from $[0, T] \times\left(\mathbf{R}^{n} \backslash \Omega\right)$ is very different in Theorems 3 and 4. In Theorem 3 the principal part of the operator is unchanged so that the basic objects of geometrical optics, sound speeds and rays, are not affected by the perturbation. What one has is very rapid dissipation with a half life of the order $1 / \lambda$ in the region $\mathbf{R}^{n} \backslash \Omega$. For the more singular problems in Theorem 4 the sound speeds $\sigma(\xi)$ are solutions of

$$
\operatorname{det}\left(\left(A_{0}+\tilde{A}_{0} \lambda\right) \sigma+\sum_{j=1}^{n} A_{j} \xi_{j}\right)=0
$$

with $|\xi|=1$. Thus outside $\Omega$ one finds that $\sigma=O(1 / \lambda)$. Waves travel very slowly outside $\Omega$.

In particular, there is a $C>0$ such that

$$
\text { support } u_{\lambda} \subset\{(t, x): \operatorname{dist}(x, \Omega) \leqslant C|t| / \lambda\} \text {. }
$$

Thus even though there may be no loss of energy in $\Omega^{c}$ the waves are effectively excluded.

6. We show by example that the condition restricting the time dependence of $\tilde{A}_{0}$ and $A_{\nu}$ cannot be entirely omitted. Consider the system

$$
\left[1+\lambda \chi_{[0, \infty)}(x)\right] P^{-2} \frac{\partial u_{\lambda}}{\partial t}+P^{-1}\left(\begin{array}{cc}
1 & 0 \\
0 & -1
\end{array}\right) P^{-1} \frac{\partial u_{\lambda}}{\partial x}-P^{-2} \frac{\partial P}{\partial t} P^{-1} u_{\lambda}=0
$$

where $P(t)$ is a nonsingular positive symmetric matrix-valued function to be specified below and $\chi_{F}$ signifies the characteristic function of $F$. Then

$$
\tilde{A}_{0}=P^{-2}, \quad A_{\nu}=P^{-1}\left(\begin{array}{cc}
1 & 0 \\
0 & -1
\end{array}\right) P^{-1}, \quad \tilde{A}_{0}^{-1 / 2} A_{\nu} \tilde{A}_{0}^{-1 / 2}=\left(\begin{array}{cc}
1 & 0 \\
0 & -1
\end{array}\right) .
$$

According to Theorem 4 the expected limiting boundary condition is that the second component of $P^{-1} u$ vanishes when $x=0$. We will show that for a suitable choice of $P$ this limiting condition is not satisfied, that is

$$
\text { second component }\left(P^{-1} u_{\lambda}(t, 0)\right) \nrightarrow 0 \quad \text { as } \lambda \rightarrow \infty .
$$

For this example the restriction that $\tilde{A}_{0}^{1 / 2} \partial\left(\tilde{A}_{0}^{-1 / 2}\right) / \partial t$ maps $M\left(\tilde{A}_{0}^{-1 / 2} A_{\nu} \tilde{A}_{0}^{-1 / 2}\right)$ into itself will be violated.

The substitution $u_{\lambda}=P v_{\lambda}$ transforms the system to

$$
\left(1+\lambda \chi_{[0, \infty)}\right) \frac{\partial v_{\lambda}}{\partial t}+\left(\begin{array}{cc}
1 & 0 \\
0 & -1
\end{array}\right) \frac{\partial v_{\lambda}}{\partial x}+\lambda \chi_{[0, \infty)} P^{-1} P_{t} v=0 .
$$

We choose

$$
P(t)=\exp \left(\begin{array}{cc}
0 & -t \\
-t & 0
\end{array}\right)
$$

so

$$
P^{-1} P_{t}=\left(\begin{array}{cc}
0 & -1 \\
-1 & 0
\end{array}\right), \quad P(0)=0
$$


Then $P=P^{*}$ and $P(0)$ is positive so we may choose $0<T$ so that $P$ is positive for $0 \leqslant t \leqslant T$.

For Cauchy data we take

$$
v_{\lambda}(0, x)=\left(\chi_{[-T, 0]}(x), 0\right) .
$$

It is not difficult to show that the solution $v_{\lambda}=\left(v_{\lambda}^{1}, v_{\lambda}^{2}\right)$ on $[0, T] \times \mathbf{R}$ satisfies

$$
\begin{aligned}
& v_{\lambda}^{i} \geqslant 0, \quad i=1,2, \\
& v^{1} \geqslant 1 \quad \text { if } x-t /(1+\lambda) \leqslant 0 \quad \text { and } t \leqslant x-T .
\end{aligned}
$$

Then, for the second component $v_{\lambda}^{2}$ in $x \geqslant 0$ we have

$$
\left[(1+\lambda) \frac{\partial}{\partial t}-\frac{\partial}{\partial x}\right] v_{\lambda}^{2}=\lambda v_{\lambda}^{1} \geqslant \lambda \chi_{[-T, 0]}(x-t /(1+\lambda)) .
$$

Integrating from time zero yields

$$
v_{\lambda}^{2}(t, 0) \geqslant \lambda t / 2(1+\lambda) \text {. }
$$

In particular, it is not true that $v_{\lambda}^{2} \rightarrow 0$ as $\lambda \rightarrow \infty$. Thus $u_{\lambda}$ provides the desired counterexample.

Notation. We will use lower case letters $e_{i j}, b_{k}$, etc. for the functions obtained after localization and change of coordinates, use $D_{j}$ for $\partial / \partial x_{j}$ in these cases and eventually use the standard summation convention instead of the sign $\Sigma$. Finally we will use the same letter $c$ for several constants, all of them independent of $\varepsilon$ and $\lambda$.

3. Basic a priori estimates and weak convergence. It is known (see [1] for example) that the solution $u_{\varepsilon}$ of the Dirichlet problem (2.7) lies in $H^{2}(\Omega) \cap \dot{H}^{1}(\Omega)$ and, for $\delta \geqslant \delta_{0}+1$, satisfies

$$
\varepsilon \delta\left\|\nabla u_{\varepsilon}\right\|_{L^{2}(\Omega)}^{2}+\delta^{2}\left\|u_{\varepsilon}\right\|_{L^{2}(\Omega)}^{2} \leqslant c\|f\|_{L^{2}(\Omega)}^{2} .
$$

Here and in all our succeeding work $c$ will be used to denote constants which are independent of $\varepsilon, \delta$ and the data of the boundary value problems, in this case $f$.

Similarly, there is a unique solution $u_{\lambda} \in L^{2}(\Omega)$ of the boundary value problem (2.9) and we have

$$
\operatorname{Re}\left(\delta u_{\lambda}+L u_{\lambda}, u\right) \geqslant\left(\delta-\delta_{0}\right) \int_{\Omega}\left|u_{\lambda}(x)\right|^{2} d x
$$

Since $P$ is strictly positive on $\Omega^{c}$ this implies that for $\delta>\delta_{0}+1$,

$$
\delta^{2}\left\|u_{\lambda}\right\|_{L^{2}\left(\mathbf{R}^{n}\right)}^{2}+\lambda \delta\left\|u_{\lambda}\right\|_{L^{2}\left(\Omega^{c}\right)}^{2} \leqslant c\|f\|_{L^{2}(\Omega)}^{2} .
$$

Finally there is a unique solution of (2.12) and (2.14). Using the relation "weak" = "strong" proved for instance in [8], one can show that this solution belongs to the space $C\left([0, T] ; L^{2}\left(\mathbf{R}^{n}\right)\right)$ and satisfies the estimate

$$
\begin{aligned}
\int_{0}^{t} \operatorname{Re}\left(L(s) u_{\lambda}(s, \cdot), u_{\lambda}(s, \cdot)\right) d s \geqslant & \frac{\delta_{1}}{2}\left\|u_{\lambda}(t, \cdot)\right\|_{L^{2}\left(\mathbf{R}^{n}\right)}^{2}-c\left\|u_{\lambda}(0, \cdot)\right\|_{L^{2}\left(\mathbf{R}^{n}\right)}^{2} \\
& +\delta_{0} \int_{0}^{t}\left\|u_{\lambda}(s, \cdot)\right\|_{L^{2}\left(\mathbf{R}^{n}\right)}^{2} d s
\end{aligned}
$$


From (3.4) we deduce that the solutions of (2.12) and (2.14) with supp $u_{\lambda}(0, \cdot) \subset \bar{\Omega}$ satisfy, for $0 \leqslant t \leqslant T$,

$$
\left\|u_{\lambda}(t, \cdot)\right\|_{L^{2}(\Omega)}^{2}+\lambda\left\|u_{\lambda}\right\|_{L^{2}\left([0, t] \times \Omega^{c}\right)}^{2} \leqslant c\left\|u_{\lambda}(0, \cdot)\right\|_{L^{2}(\Omega)}^{2}+c\|f\|_{L^{2}\left([0, t] \times \mathbf{R}^{n}\right)}^{2} .
$$

The last problem (2.13)-(2.14) requires some changes in the underlying theory so we review some of the basic facts about scar problems. As pointed out in the introduction $\left(L+\lambda \tilde{A}_{0}(\partial / \partial t)\right) u$ is not a well-defined distribution for arbitrary $u \in$ $L_{2}\left([0, T] \times \mathbf{R}^{n}\right)$. One way to avoid this problem is to write $\left(L+\lambda \tilde{A}_{0}(\partial / \partial t)\right) u$ in the divergence form

$$
\frac{\partial}{\partial t}\left(A_{0}+\lambda \tilde{A}_{0}\right) u_{\lambda}+\sum \frac{\partial}{\partial x_{j}}\left(A_{j} u_{\lambda}\right)+\left(B-\frac{\partial A_{0}}{\partial t}-\lambda \frac{\partial \tilde{A}_{0}}{\partial t}-\sum_{j} \frac{\partial A_{j}}{\partial x_{j}}\right) u_{\lambda} .
$$

This expression does have meaning for $u \in L^{2}\left([0, T] \times \mathbf{R}^{n}\right)$, and if it lies in $L^{2}\left([0, T] \times \mathbf{R}^{n}\right)$ then $\left.\left(A_{0}+\lambda \tilde{A}_{0}\right) u\right|_{t=0}$ is a well-defined element of $H_{-1 / 2}\left(\mathbf{R}^{n}\right)$ so the initial condition (2.14) makes sense. An equivalent method (and the one proposed by Friedrichs) is the following:

Let

$$
\begin{aligned}
& S=[0, T] \times \mathbf{R}^{n}, \quad S_{\omega}=[0, T] \times \omega \quad \text { for any } \omega \subset \mathbf{R}^{n} ; \\
& \theta=\left\{u \in \mathcal{E}^{\prime}\left(\mathbf{R}^{n}\right) \mid u \in C^{\infty}\left(S_{\bar{\Omega}}\right) \cap C^{\infty}\left(S_{\Omega^{c}}\right) \text { and } A_{\nu} u\right. \text { is continuous } \\
& \quad \text { on a neighborhood of }[0, T] \times \partial \Omega\} .
\end{aligned}
$$

Functions in $\theta$ are smooth up to $\partial \Omega$ from both sides and $A_{\nu} u$ is continuous across $\partial \Omega$. We say that $u \in L^{2}(S)$ is a weak solution of (2.13) and (2.14) iff ( $\forall v \in \theta$ with $v(T)=0)$

$$
\left(u,\left(L^{*} v-\lambda \frac{\partial}{\partial t} \tilde{A}_{0} v\right)\right)_{S}=(f, v)_{S}+\left(g,\left(A_{0}+\lambda \tilde{A}_{0}\right) v(0, \cdot)\right)_{\mathbf{R}^{n}} .
$$

The basic theorem is the following.

Friedrich's TheOREM [4]. For any $f \in L^{2}(S)$ and $g \in L^{2}\left(\mathbf{R}^{n}\right)$, there is a unique weak solution $u_{\lambda}$ of (2.13) and (2.14). In addition, $u_{\lambda} \in C\left([0, T] ; L_{2}\left(\mathbf{R}^{n}\right)\right)$ and there is a constant $c$ independent of $f, g, t \in[0, T]$, and $\lambda \geqslant 0$ such that

$$
\lambda\left\|u_{\lambda}(t)\right\|_{L^{2}\left(\Omega^{c}\right)}^{2}+\left\|u_{\lambda}(t)\right\|_{L^{2}\left(\mathbf{R}^{n}\right)}^{2} \leqslant c\left(\|f\|_{L^{2}(S)}^{2}+\|g\|_{L^{2}\left(\mathbf{R}^{n}\right)}^{2}+\lambda\|g\|_{L^{2}\left(\Omega^{c}\right)}^{2}\right) .
$$

SKETCH OF PROOF. For $u \in \theta$ we can prove (3.6) by taking the scalar product (in $\left.L^{2}([0, t] \times \Omega)\right)$ of $(3.2)$ with $u$ and integrate by parts to obtain

$$
\left.\left(A_{0} u, u\right)_{\Omega}\right|_{0} ^{t}=\operatorname{Re}(u, f)_{[0, t] \times \Omega}+\left(A_{\nu} u, u\right)_{[0, t] \times \partial \Omega}+(Z u, u)_{[0, t] \times \Omega}
$$

where the boundary integral involves the limits of $u$ on $\partial \Omega$ from $\Omega$ and $2 Z=\partial A_{0} / \partial t$ $-B-B^{*}+\sum_{j} \partial A_{j} / \partial x_{j}$. Similarly on $[0, t] \times \Omega^{c}$ we have

$$
\begin{aligned}
\left.\left(\left(A_{0}+\lambda \tilde{A}_{0}\right) u, u\right)_{\Omega^{c}}\right|_{0} ^{t}= & \operatorname{Re}(u, f)_{[0, t] \times \Omega^{c}}-\left(A_{\nu} u, u\right)_{[0, t] \times \partial \Omega} \\
& +\left(\left(Z+\frac{\lambda}{2} \frac{\partial \tilde{A}_{0}}{\partial t}\right) u, u\right)_{[0, t] \times \Omega^{c}}
\end{aligned}
$$


where the boundary term involves the limits of $u$ from $\Omega^{c}$. However, since $A_{\nu} u$ is continuous across $\partial \Omega$ the boundary integrals cancel when one adds these expressions. The positivity of $A_{0}$ and $\tilde{A}_{0}$ yields (3.6) after an application of the Schwarz and Gronwall inequalities.

A similar "adjoint" inequality implies that $\forall v \in \theta$ with $v(T)=0$ we have

$$
\|v(0)\|_{L^{2}\left(\mathbf{R}^{n}\right)}+\|v\|_{L^{2}(S)} \leqslant c\left\|L^{*} v-\lambda \frac{\partial}{\partial t} \tilde{A}_{0} v\right\|_{L^{2}(S)} .
$$

Let $\Gamma=\left\{L^{*} v-\lambda(\partial / \partial t) \tilde{A}_{0} v \mid v \in \theta\right.$ and $\left.v(T)=0\right\}$; then the existence of a weak solution follows from an application of the Riesz Representation Theorem to the linear functional $\xi: \Gamma \rightarrow \mathbf{C}$ defined by

$$
L^{*} v-\lambda \frac{\partial}{\partial t} \tilde{A}_{0} v \stackrel{\xi}{\mapsto}\left(\left(A_{0}+\lambda \tilde{A}_{0}\right) v(0), g\right)+(v, f) .
$$

The next step is to show that if $u_{\lambda}$ is a weak solution the there exist $u_{\lambda}^{m} \in \theta$ such that as $m \rightarrow \infty$,

$$
\begin{aligned}
u_{\lambda}^{m} & \rightarrow u_{\lambda} \quad \text { in } L^{2}(S), \\
\left(L+\lambda \tilde{A}_{0} \frac{\partial}{\partial t}\right) u_{\lambda}^{m} & \rightarrow f \quad \text { in } L^{2}(S), \quad \text { and } \\
u_{\lambda}^{m}(0, \cdot) & \rightarrow g \quad \text { in } L^{2}\left(\mathbf{R}^{n}\right) .
\end{aligned}
$$

This "weak = strong" result is proved with mollifiers as in [8], [11]. Inequality (3.6) shows that $u_{\lambda}^{1}, u_{\lambda}^{2}, \ldots$ is a Cauchy sequence in $C\left([0, T] ; L^{2}\left(\mathbf{R}^{n}\right)\right)$. It follows that $u_{\lambda} \in C\left([0, T] ; L^{2}\left(\mathbf{R}^{n}\right)\right)$ and that inequality (3.6) holds for $u_{\lambda}$. In particular, there is at most one solution.

We turn next to a discussion of the weak convergence of the solutions as $\varepsilon \rightarrow 0$ or $\lambda \rightarrow \infty$. In [1] it has been shown that when $\varepsilon$ goes to zero, the solution $u_{\varepsilon}$ of $(2.7)$ converges weakly in $L^{2}(\Omega)$, to the solution of (2.8). Similarly, the solution of (2.9), ((2.12), (2.13)) and ((2.12), (2.14)) are uniformly bounded (with respect to $\lambda$ ) in $L^{2}\left(\mathbf{R}^{n}\right)$ and $C\left([0, T] ; L^{2}\left(\mathbf{R}^{n}\right)\right)$, respectively. Therefore, there exist some subfamily of functions (still denoted $u_{\lambda}$ ) which converges in $L^{2}\left(\mathbf{R}^{n}\right)$ weak, respectively $L^{\infty}\left([0, T] ; L^{2}\left(\mathbf{R}^{n}\right)\right)$ weak star, to a limit $u$. The a priori estimates (3.3), (3.5) and (3.6) imply that in the case of Theorems 2,3 and 4 we have, respectively,

$$
\begin{gathered}
\int_{\Omega^{c}}\left|u_{\lambda}\right|^{2} d x \leqslant \frac{C}{\lambda}, \quad \int_{0}^{T} \int_{\Omega^{c}}\left|u_{\lambda}\right|^{2} d x d t \leqslant \frac{C}{\lambda}, \quad \text { and } \\
\sup _{0 \leqslant t \leqslant T} \int_{\Omega}\left|u_{\lambda}(t, x)\right|^{2} d x \leqslant \frac{C}{\lambda} .
\end{gathered}
$$

Therefore, in the case of Theorem $2,\left.u(x)\right|_{\Omega^{c}}=0$, and $\left.u(t, x)\right|_{[0, T] \times \Omega^{c}}=0$ in case of Theorems 3 and 4. Furthermore, in the sense of distributions we have

$$
\begin{aligned}
\delta u+L u & =f \quad \text { in } \Omega \quad(\text { Theorem 2), and } \\
L u & =f \quad \text { in }[0, T] \times \Omega \quad \text { (Theorems } 3 \text { and 4). }
\end{aligned}
$$

The main point will be to prove that $u$ satisfies the boundary conditions given by (2.18), (2.11), (2.16) and (2.17). Since the solutions of the resulting boundary value 
problems are unique we conclude that not only the subfamily but the whole family converges weakly. This result has been proved for the case of Theorem 1 in [1]. We will give a detailed proof in the case of Theorem 2 and give some indications of how to adapt it for Theorems 3 and 4.

We introduce a neighborhood $\theta$ of $\partial \Omega$ such that $A_{\nu}$ and all the boundary spaces are defined and smooth on $\theta$. Next we choose an open cover $\theta_{l}: l=1, \ldots, N$ of $\partial \Omega$ with $\bar{\theta}, \subset \mathcal{O}$ and so that there are diffeomorphisms

$$
\tau_{1}: \vartheta_{l} \rightarrow \mathscr{B} \equiv\left\{x \in \mathbf{R}^{n}|| x \mid<1\right\}
$$

with

$$
g t_{l}\left[\mathcal{O}_{l} \cap \Omega\right]=\mathscr{B}_{-} \equiv\left\{x \in \mathscr{B}_{\mid} \mid x_{1}<0\right\}
$$

and

$$
\left(\tau_{l}\right)_{*}(\partial / \partial \nu)=D_{1} .
$$

Choose $0 \leqslant \chi_{1} \in C_{0}^{\infty}\left(\theta_{l}\right)$ such that $\Sigma \chi_{l} \leqslant 1$ and $\Sigma \chi_{l}=1$ on a neighborhood of $\partial \Omega$. Choose $\chi_{0} \in C^{\infty}\left(\mathbf{R}^{n}\right)$ such that $\chi_{0}=0$ on a neighborhood of $\partial \Omega$ and $\chi_{0}=1$ on a neighborhood of $\left\{x \mid \Sigma \chi_{l}(x)<1\right\}$. For $l \geqslant 1$, let $u_{\lambda}^{l}=\left(\chi_{l} u_{\lambda}\right) \circ \tau_{l}^{-1} \in L^{2}(\Re)$ be the localization of $u$ to $\theta_{l}$ in the coordinates induced by $\tau_{l}$. For $u_{\lambda}^{\prime}$ we have the differential equations

$$
\left\{\begin{array}{l}
\left(\sum a_{k}(x) D_{k}+b(x)+\lambda p+\delta I\right) u_{\lambda}^{l}=g_{l}^{+} \quad \text { in } \mathbf{R}_{+}^{n}, \\
\left(\sum a_{k}(x) D_{k}+b(x)+\delta I\right) u_{\lambda}^{l}=g_{l}^{-} \quad \text { in } \mathbf{R}_{-}^{n}
\end{array}\right.
$$

where $a_{k}, b, p$ are matrix-valued functions defined on $\mathbf{R}^{n}$, with smoothness, symmetry and positivity properties analogous to $A_{k}, B, P$. In addition, $a_{1}=A_{\nu} \circ \tau_{l}^{-1}$ since $\left(\tau_{l}\right)_{*} \circ(\partial / \partial \nu)=D_{1}$. Henceforth we will omit the index $l$, and extend $u_{\lambda}^{l}$ and $g_{l}^{ \pm}$by zero outside the ball $|x|<1$. These functions are uniformly bounded in $L^{2}\left(\mathbf{R}^{n}\right)$. Now we put $v_{\lambda}=p^{1 / 2} u_{\lambda}$, so $v$ is a solution of the following equation. ${ }^{3}$

$$
\left(p^{-1 / 2} a_{1} p^{-1 / 2} D_{1}+\sum_{k>1} \tilde{a}_{k} D_{k}+\lambda \tilde{P}\right) v_{\lambda}=\tilde{g}
$$

where $\tilde{P}(x)=0$ if $x \in \mathbf{R}_{-}^{n}$ and $\tilde{P}(x)=I$ if $x \in \mathbf{R}_{+}^{n}$, and where $\tilde{g}$ denotes a function bounded in $L^{2}\left(\mathbf{R}^{n}\right)$ uniformly in $\lambda$. If $K_{-}$denotes the orthogonal projector on the negative subspace of the matrix $p^{-1 / 2} a_{1} p^{-1 / 2}$, then $\partial\left(K_{-} v_{\lambda}\right) / \partial x_{1}$ belongs to the space $L^{2}\left(\mathbf{R} ; H^{-1}\left(\mathbf{R}^{n-1}\right)\right)$ and, therefore, $\left(K_{-} v_{\lambda}\right)(0, \cdot)$ is defined (at least in $\left.H^{-1 / 2}\left(\mathbf{R}^{n-1}\right)\right)$. Furthermore, $\left.D_{1}\left(K_{-} v_{\lambda}\right)\right|_{\mathbf{R}_{-}}$is uniformly (with respect to $\lambda$ ) bounded in $L^{2}\left(\mathbf{R}_{-} ; H^{-1}\left(\mathbf{R}^{n-1}\right)\right)$, and $K_{-} v_{\lambda}$ is uniformly bounded in $L^{2}\left(\mathbf{R}_{-} ; L^{2}\left(\mathbf{R}^{n-1}\right)\right)$. Therefore, introducing a new subfamily we deduce that we have:

$$
\begin{gathered}
v_{\lambda} \rightarrow v \quad \text { in } L^{2}\left(\mathbf{R}_{-} ; L^{2}\left(\mathbf{R}^{n-1}\right)\right), \\
K_{-} v_{\lambda}(0, \cdot) \rightarrow K_{-} v(0, \cdot) \quad \text { in } H^{-1 / 2}\left(\mathbf{R}^{n-1}\right) .
\end{gathered}
$$

${ }^{3}$ For $x_{1}<0$ we put $p\left(x_{1}, \hat{x}\right)=p\left(-x_{1}, \hat{x}\right)$. 
From (3.10) we deduce that $u_{\lambda} \rightarrow \tilde{u} \equiv p^{-1 / 2} v$ in $L^{2}(B)$ and $\left.K_{-} p^{1 / 2} u_{\lambda}\right|_{x_{1}=0}$ converges to $\left.K_{-} p^{1 / 2} \tilde{u}\right|_{x_{1}=0}$ in $H^{-1 / 2}\left(\mathbf{R}^{n-1}\right)$. Finally we will show that $\left.K_{-} p^{1 / 2} \tilde{u}\right|_{x_{1}=0}=0$ or, equivalently, $K_{-} v(0, \cdot)=0$. It suffices to prove that $K_{-} v_{\lambda}(0, \cdot)$ converges to zero in $H^{-1}\left(\mathbf{R}^{n-1}\right)$. In $\mathbf{R}_{+}^{n}$ we have

$$
K_{-} p^{-1 / 2} a_{1} p^{-1 / 2} K_{-} D_{1}\left(K_{-} v_{\lambda}\right)+\lambda K_{-} v_{\lambda}=h_{\lambda}
$$

where $h_{\lambda}$ is uniformly bounded in the space $L^{2}\left(\mathbf{R}_{+}, H^{-1}\left(\mathbf{R}^{n-1}\right)\right)$. The operator $K_{-} p^{-1 / 2} a_{1} p^{-1 / 2} K_{-}$restricted to $K_{-}\left(\mathbf{C}^{k}\right)$ is strictly negative. Therefore we deduce from (3.11) that we have (with a different $h_{\lambda}$ )

$$
-D_{1}\left(K_{-} v_{\lambda}\right)+\lambda \beta K_{-} v_{\lambda}=h_{\lambda} \text { in } \mathbf{R}_{+} \times \mathbf{R}^{n-1}
$$

where $\beta$ is a smooth and strictly positive matrix. Denote by $\hat{\Delta}$ the Laplacian in the tangential variables $x_{2}, x_{3}, \ldots, x_{n}$. For $\mu>0, \mu-\hat{\Delta}$ defines an isomorphism of $L^{2}\left(\mathbf{R}_{+} ; H^{1}\left(\mathbf{R}^{n-1}\right)\right)$ onto $L^{2}\left(\mathbf{R}_{+} ; H^{-1}\left(\mathbf{R}^{n-1}\right)\right)$. Define $w_{\lambda}$ by $K_{-} v_{\lambda}=(\mu-\hat{\Delta}) w_{\lambda}$. Multiply both sides of (3.12) by $w_{\lambda} d x_{2} d x_{3} \ldots d x_{n}$ and integrate over $\mathbf{R}^{n-1}$ to obtain

$$
\begin{aligned}
& \left(h_{\lambda}, w_{\lambda}\right)_{L^{2}\left(\mathbf{R}^{n-1}\right)} \\
& \quad=\left(\lambda \beta K_{-} v_{\lambda}, w_{\lambda}\right)_{L^{2}\left(\mathbf{R}^{n-1}\right)}-D_{1}\left(K_{-} v_{\lambda},(\mu-\hat{\Delta})^{-1} K_{-} v_{\lambda}\right)_{L^{2}\left(\mathbf{R}^{n-1}\right)}
\end{aligned}
$$

It is not difficult to see that for $\mu$ sufficiently large one has

$$
\left(\lambda \beta K_{-} v_{\lambda}, w_{\lambda}\right)_{L^{2}\left(\mathbf{R}^{n-1}\right)}=\left(\lambda \beta(\mu-\hat{\Delta}) w_{\lambda}, w_{\lambda}\right)_{L^{2}\left(\mathbf{R}^{n-1}\right)} \geqslant \lambda \gamma\left\|w_{\lambda}\right\|_{H^{1}\left(\mathbf{R}^{n-1}\right)}^{2}
$$

where $\gamma$ is a strictly positive constant. Thus

$$
-\frac{1}{2} D_{1}\left\|w_{\lambda}\right\|_{H^{1}\left(\mathbf{R}^{n-1}\right)}^{2}+\lambda \gamma\left\|w_{\lambda}\right\|_{H^{1}\left(\mathbf{R}^{n-1}\right)}^{2} \leqslant\left(h_{\lambda}, w_{\lambda}\right)_{L^{2}\left(\mathbf{R}^{n-1}\right)}
$$

where the right-hand side is bounded in $L^{1}\left(\mathbf{R}_{+}\right)$since $h_{\lambda}$ is bounded in $L^{2}\left(\mathbf{R}_{+} ; H^{-1}\left(\mathbf{R}^{n-1}\right)\right)$ and $w_{\lambda}$ is bounded in $L^{2}\left(\mathbf{R}_{+} ; H^{1}\left(\mathbf{R}^{n-1}\right)\right)$. Integrating (3.14) over $\mathbf{R}_{+}$yields

$$
\frac{1}{2}\left\|w_{\lambda}(0, \cdot)\right\|_{H^{1}\left(\mathbf{R}^{n-1}\right)}^{2}+\lambda \gamma\left\|w_{\lambda}\right\|_{L^{2}\left(\mathbf{R}_{+} ; H^{1}\left(R^{n-1}\right)\right)}^{2} \leqslant c\left\|w_{\lambda}\right\|_{L^{2}\left(R_{+} ; H^{1}\left(\mathbf{R}^{n-1}\right)\right)}
$$

which implies that

$$
\left\|w_{\lambda}\right\|_{L^{2}\left(\mathbf{R}_{+} ; H^{1}\right)}=O\left(\lambda^{-1}\right) \quad \text { and } \quad\left\|w_{\lambda}(0, \cdot)\right\|_{H^{1}\left(\mathbf{R}^{n-1}\right)}=O\left(\lambda^{-1 / 2}\right)
$$

as $\lambda \rightarrow \infty$. Thus, $\left\|K_{-} v_{\lambda}(0, \cdot)\right\|_{H^{-1}\left(\mathbf{R}^{n-1}\right)} \leqslant c\left\|w_{\lambda}(0, \cdot)\right\|_{H^{1}\left(\mathbf{R}^{n-1}\right)}$ goes to zero when $\lambda$ goes to infinity, so $\left.K_{-} p^{1 / 2} u_{\lambda}\right|_{x_{1}=0}$ converges to zero on $H^{-1 / 2}\left(\mathbf{R}^{n-1}\right)$, and the proof of weak convergence in Theorem 2 is complete.

The proof of the weak convergence in case of Theorem 3 is similar; in fact, the operator $L(t)=A_{0}(t, x)(\partial / \partial t)+\sum A_{j}(t, x)\left(\partial / \partial x_{j}\right)+B+\lambda P$ defined in $L^{2}\left([0, T] \times \mathbf{R}^{n}\right)$ is of the same type as the operator $\sum A_{j}\left(\partial / \partial x_{j}\right)+B+\lambda P$ defined in $L^{2}\left(\mathbf{R}^{n}\right)$. The only difference is that the domain $[0, T] \times \Omega$ has a corner and that the boundary condition is not of constant rank there. If $\bar{u}$ is a weak limit point in $L^{2}([0, T] \times \Omega)$ of $\left.u_{\lambda}\right|_{[0, T] \times \Omega}$ we must show that $\bar{u}$ is a weak solution of the boundary value problem (2.16). Precisely we need to show that

$$
\iint_{[0, T] \times \Omega}\left\langle\bar{u}, L^{*} \varphi\right\rangle-\langle f, \varphi\rangle d t d x=\int_{\Omega}\langle g, \varphi\rangle d x
$$


for all $\varphi \in C_{(0)}^{1}([0, T] \times \Omega)$ such that $\varphi(T)=0$, and $\varphi$ satisfies the adjoint boundary condition

$$
\varphi \perp A_{\nu} P^{-1 / 2} M\left(P^{-1 / 2} A_{\nu} P^{-1 / 2}\right) \text { on }[0, T] \times \partial \Omega .
$$

Let $\psi_{\varepsilon}$ be a simple cut off function vanishing at points less than $\varepsilon$ units of length from $\{t=0$ or $t=T\} \times \partial \Omega$ and equal to one at points at last $2 \varepsilon$ units from the same corners. We may choose $\psi_{\varepsilon}$ with $\nabla \psi_{\varepsilon}=O(1 / \varepsilon)$. In this case replacing $\varphi$ by $\psi_{\varepsilon} \varphi$ changes each term in the above identity by a quantity $o(1)$ as $\varepsilon \rightarrow 0$ so it suffices to prove the identity with $\varphi$ replaced by $\psi_{\varepsilon} \varphi$ and then to pass to the limit $\varepsilon \rightarrow 0$. With $\varphi$ replaced by $\psi_{\varepsilon} \varphi$, it suffices to study the behavior of $u_{\lambda}$ and $\bar{u}$ on small neighborhoods of point $(t, x)$ such that $x \in \partial \Omega, t \neq 0$ and $t \neq T$, or $x \in \Omega, t=0$ or $t=T$. In these neighborhoods the analysis proceeds as before.

The weak convergence in Theorem 4 uses the same tools but is somewhat more difficult. To make the going a little easier we observe that because of the bound (3.6) it suffices to prove the weak convergence for a set of $f, g$ dense in $L^{2}([0, T] \times \Omega)$ and $L^{2}(\Omega)$, respectively. For example we may suppose $f \in$ $C_{0}^{\infty}([0, T] \times \Omega)$ and $g \in C_{0}^{\infty}(\Omega)$. In this case we will shortly prove in part (4) of Theorem 5 that $u^{\lambda}$ has additional tangential regularity, uniformly in $\lambda$. Using the same localization in $x$ the analogue of equation (3.8) is

$$
\left(a_{0}(t, x)+\lambda \tilde{a}_{0}(t, x)\right) \frac{\partial u_{\lambda}^{l}}{\partial t}+\sum_{i=1}^{n} a_{i}(t, x) D_{i} u_{\lambda}+b u_{\lambda}^{l}=g_{l}
$$

with $u_{\lambda}^{l}$ defined in $\mathbf{R}_{+} \times \mathbf{R}^{n}$ with support in the open set $|x|<1$, and with the function $\tilde{a}_{0}(t, x)$ satisfying the hypothesis

$$
\tilde{a}_{0}(t, x)=0 \quad \text { if } x_{1}<0, \quad \tilde{a}_{0}(t, x)>\delta I \quad \text { if } x_{1}>0 .
$$

Using the tangential regularity we see that in $[0, T] \times\left\{x_{1}>0\right\}, \lambda \tilde{a}_{0}\left(\partial u_{\lambda}^{l} / \partial t\right)+$ $a_{1} D_{1} u_{\lambda}^{l}$ lies in a bounded subset of $L^{2}\left([0, T] \times\left\{x_{1} \geqslant 0\right\}\right)$ for $\lambda \geqslant \lambda_{0}$. Letting $v_{\lambda}=\tilde{a}_{0}^{1 / 2} u_{\lambda}^{l}$ for $x_{1}>0$ we find that for $\lambda$ large

$$
\lambda \frac{\partial v_{\lambda}}{\partial t}+\tilde{a}_{0}^{-1 / 2} a_{1} \tilde{a}_{0}^{-1 / 2} D_{1} v_{\lambda}+\lambda \tilde{a}_{0}^{-1 / 2} \frac{\partial \tilde{a}_{0}^{-1 / 2}}{\partial t} v_{\lambda}
$$

lies in a bounded subset of $L^{2}\left([0, T] \times\left\{x_{1} \geqslant 0\right\}\right)$. Let $K_{-}(t, x)$ be the orthogonal projector onto the negative eigenspace of $\tilde{a}_{0}^{-1 / 2} a_{1} \tilde{a}_{0}^{-1 / 2}$. Taking the $\mathbf{C}^{k}$ scalar product (3.17) with $K_{-} v_{\lambda}$ we find that

$$
\begin{aligned}
& \frac{\lambda}{2} \frac{\partial}{\partial t}\left|K_{-} v_{\lambda}\right|^{2}+D_{1}\left\langle K_{-} v_{\lambda}, \tilde{a}_{0}^{-1 / 2} a_{1} \tilde{a}_{0}^{-1 / 2} K_{-} v_{\lambda}\right\rangle \\
& +\lambda\left\langle K_{-} v_{\lambda}, \tilde{a}_{0}^{-1 / 2} \frac{\partial \tilde{a}_{0}^{-1 / 2}}{\partial t} v_{\lambda}\right\rangle+2 \lambda \operatorname{Re}\left\langle v_{\lambda}, K_{-} \frac{\partial K_{-}}{\partial t} v_{\lambda}\right\rangle
\end{aligned}
$$

lies in a bounded subset of $L^{1}\left([0, T] \times\left\{x_{1} \geqslant 0\right\}\right)$. Letting $K_{+}=I-K_{-}$the last two terms in (3.18) are written as

$$
\lambda\left\langle K_{-} v_{\lambda}, \tilde{a}_{0} \frac{\partial \tilde{a}_{0}^{-1 / 2}}{\partial t} K_{-} v_{\lambda}\right\rangle+\lambda\left\langle K_{-} v_{\lambda}, K_{-} \tilde{a}_{0}^{-1 / 2} \frac{\partial \tilde{a}_{0}^{-1 / 2}}{\partial t} K_{+} v_{\lambda}\right\rangle
$$


and

$$
2 \lambda \operatorname{Re}\left\langle K_{-} v_{\lambda}, \frac{\partial K_{-}}{\partial t} K_{-} v_{\lambda}\right\rangle+2 \lambda \operatorname{Re}\left\langle K_{-} v_{\lambda}, K_{-} \frac{\partial K_{-}}{\partial t} K_{+} v_{\lambda}\right\rangle .
$$

The first term of each of these is no larger than $c \lambda\left|K_{-} v_{\lambda}\right|^{2}$. It is in estimating the last terms in (3.19), (3.20) that we use the restrictions on the time dependence of $\tilde{A}_{0}$ and $A_{\nu}$. As observed in (2.18) the $v_{\lambda}$ vanish for $x_{1} \geqslant c / \lambda$ and the matrices $\tilde{a}_{0}$, $\partial \tilde{a}_{0}^{-1 / 2} / \partial t, K_{-}$, and $\partial K_{-} / \partial t$ at the point $\left(t, x, x_{1}, \ldots, x_{n}\right)$ differ from their values at $\left(t, 0, x_{1}, \ldots, x_{n}\right)$ by quantities $O(1 / \lambda)$. On the other hand, the restriction on the time dependence of the coefficients implies that

$$
\left.K_{-} \tilde{a}_{0}^{-1 / 2} \frac{\partial \tilde{a}_{0}}{\partial t} K_{+}\right|_{\left(t, 0, x^{\prime}\right)}=0 \text { and }\left.K_{-} \frac{\partial K_{-}}{\partial t} K_{+}\right|_{\left(t, 0, x^{\prime}\right)}=0 \text {. }
$$

Thus the integrals of (3.18) and (3.19) over $[0, t] \times\left\{x_{1}>0\right\}$ are bounded by

$$
c \lambda \int_{0}^{t} \int_{x_{1} \geqslant 0}\left|K_{-} v_{\lambda}\right|\left(\left|K_{-} v_{\lambda}\right|+\frac{1}{\lambda}\left|v_{\lambda}\right|\right) d t d x .
$$

To take advantage of this we set

$$
Q_{\lambda}(t) \equiv\left(\int_{x_{1} \geqslant 0}\left|K_{-}(t, x) v_{\lambda}(t, x)\right|^{2} d x\right)^{1 / 2}
$$

Note that $Q(0)=0$. Integrating (3.18) over $[0, t] \times\left\{x_{1} \geqslant 0\right\}$ then yields

$$
\begin{gathered}
\lambda Q_{\lambda}^{2}(t)-\int_{0}^{t} \int_{\left\{x_{1}=0\right\}}\left\langle\tilde{a}_{0}^{-1 / 2} a_{1} \tilde{a}_{0}^{-1 / 2} K_{-} v_{\lambda}, K_{-} v_{\lambda}\right\rangle d t d x_{2} \ldots d x_{n} \\
\leqslant c \lambda \int_{0}^{t} Q_{\lambda}^{2}(s) d s+c \int_{0}^{t} Q(s) d s
\end{gathered}
$$

Notice that

$$
\left\langle\tilde{a}_{0}^{-1 / 2} a_{1} \tilde{a}_{0}^{-1 / 2} K_{-} v_{\lambda}, K_{-} v_{\lambda}\right\rangle \leqslant-C\left|K_{-} v_{\lambda}\right|^{2}
$$

so the integral on the left of (3.21) is negative. Applying Gronwall's method to the remaining terms one finds that $Q_{\lambda} \leqslant C t / \lambda$ for $0 \leqslant t \leqslant T$. Notice that the basic energy estimate (3.6) shows that $Q_{\lambda} \leqslant C / \sqrt{\lambda}$ so the $K_{-} v_{\lambda}$ components are smaller than $v_{\lambda}$ itself. Using this refined estimate for $Q_{\lambda}$ in equation (3.21) we find

$$
-\left.\int_{0}^{t} \int\left\langle\tilde{a}_{0}^{-1 / 2} a_{\mathrm{r}} \tilde{a}_{0}^{-1 / 2} K_{-} v_{\lambda}, K_{-} v_{\lambda}\right\rangle\right|_{x_{1}=0} d x_{2} \ldots d x_{n} d t \leqslant c t / \lambda .
$$

Because of (3.22) this implies that

$$
\left\|K_{-} \tilde{a}_{0}^{1 / 2} u_{\lambda}^{l}\right\|_{L^{2}\left([0, T] \times\left\{x_{1} \geqslant 0\right\}\right)}=O\left(\lambda^{-1 / 2}\right)
$$

and the proof is complete.

4. Tangential regularity. The main goals of this section are to show that as $\varepsilon \rightarrow 0$ or $\lambda \rightarrow \infty$ the "tangential derivatives" of the solutions to our problems can be estimated independent of $\varepsilon$ and $\lambda$.

The Dirichlet problem (2.7) is best known. If $f \in H_{s}(\Omega)$ for $-1 \leqslant s \leqslant 1$ then $u_{\varepsilon} \in H_{s+2}(\Omega) \cap \stackrel{\circ}{H}_{1}$. The restriction $|s| \leqslant 1$ arises from the fact that the coefficients 
need not be infinitely smooth. As $\varepsilon \rightarrow 0$ the derivatives of $u_{\varepsilon}$ in the directions normal to $\partial \Omega$ become large. However, some tangential regularity persists.

For the other problems, the solutions need not be smooth when the data are smooth. The main point is that $\partial \Omega$ is allowed to be characteristic for the differential operator $L$ ( $A_{\nu}$ may have nontrivial kernel). It is well known that this may cause a "loss of derivatives in the normal directions." Nevertheless, solutions will be smooth in the tangential directions and this regularity will persist in the limit $\lambda \rightarrow \infty$.

To make these remarks precise we define a space of functions whose tangential derivatives of first order are square integrable.

Definition. A vector field $\Lambda \in C^{\infty}\left(\bar{\Omega} ; \mathbf{C}^{n}\right)$ is tangential iff $\langle\Lambda(x), \nu(x)\rangle=0$ for all $x \in \partial \Omega$. We say that $u \in L^{2}(\Omega)$ is in $H_{\tan }^{1}(\Omega)$ if

$$
u \in H^{1}(\{x \in \Omega: \operatorname{dist}(x, \partial \Omega)>1\})
$$

and $\Lambda u \in L_{\text {loc }}^{2}(\Omega)$ for all tangential vector fields $\Lambda$.

Observe that if $\varphi \in C^{\infty}(\bar{\Omega}), \varphi=0$ on $\partial \Omega$, then for any vector field $\Gamma, \varphi \Gamma$ is tangential so $\varphi \Gamma u$ is square integrable near $\partial \Omega$ if $u \in H_{\tan }^{1}$. Thus if $u \in H_{\tan }^{1}$ then $\operatorname{dist}(x, \partial \Omega) u \in H_{\text {loc }}^{1}(\Omega)$ so the rate at which nontangential derivatives may grow at $\partial \Omega$ is restricted. The next lemma shows that the above remarks are reversible.

LEMMA 1. If $u \in L^{2}(\Omega)$ then $u \in H_{\tan }^{1}(\Omega)$ if and only if the following conditions hold:

(1) $u \in H^{1}\{x \mid \operatorname{dist}(X, \partial \Omega)>1\}$.

(2) For any $x \in \partial \Omega$ there are $k-1$ tangential vector fields $\Lambda^{1}, \Lambda^{2}, \ldots, \Lambda^{k-1}$ linearly independent at $x$ and so that $\Lambda^{i} u \in L_{\mathrm{loc}}^{2}(\Omega)$ for $i=1,2, \ldots, k-1$.

(3) For any $x \in \partial \Omega$ there is a neighborhood $\Re$ of $x$ in $\mathbf{R}^{n}$ and $\varphi \in C^{\infty}(\mathcal{X})$ so that $\left.\varphi\right|_{\partial \Omega \cap \Re}=0, \nabla \varphi(x) \neq 0$ and $\varphi u \in H^{1}(\Re \cap \Omega)$.

PROOF. The only if part is demonstrated by the remarks preceding the lemma. For the if part it suffices to prove that, for every tangential vector field $\Lambda, \Lambda u$ is square integrable on a neighborhood of each $x \in \partial \Omega$. Given such an $x$ and $\Lambda$ we may

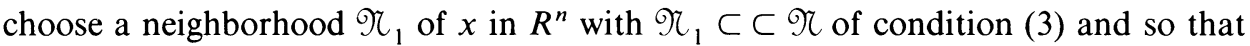
$\Lambda^{1}, \Lambda^{2}, \ldots, \Lambda^{k-1}, \nu$ form a basis of $\mathbf{C}^{k}$ for each $x \in \Re_{1}$, and $\varphi(x) \neq 0$ for $x \in$ $\Re_{1} \backslash \partial \Omega$. Then there are functions $\alpha_{j} \in C^{\infty}\left(\Re_{1}\right)$ such that

$$
\Lambda=\alpha_{0} \nu+\sum \alpha_{j} \Lambda^{j}
$$

and $\left.\alpha_{0}\right|_{\Re_{1} \cap \partial \Omega}=0$. It follows that $\alpha_{0} \varphi^{-1} \in C^{\infty}\left(\Re_{1}\right)$ so $|\Lambda u| \leqslant c|\varphi \nu u|+c \Sigma_{j}\left|\Lambda^{j} u\right|$ is square integrable near $x$ since $\varphi \nu$ and $\Lambda^{j}$ are tangential.

This lemma allows us to norm $H_{\tan }^{1}(\Omega)$ as follows. Let $\theta_{l}, \tau_{l}, \chi_{l}$ be the covering of $\Omega$, diffeomorphisms, and cut off functions introduced in $\S 2$. As before, $u^{l}=$ $\left(\chi_{l} u\right) \circ \tau_{l}^{-1}$ for $l>0$ so that $u^{l}$ has as its natural domain $\mathscr{B}_{-}=\{x:|x|<1$ and $\left.x_{1}<0\right\}$ with $\partial \Omega$ corresponding to $x_{1}=0$. The tangential vector fields which we choose are given in local coordinates by $D_{k}, k=2, \ldots, n$, and the function $\varphi$ is $x_{1}$. Then

$$
\begin{gathered}
\|u\|_{H_{\tan }^{1}}^{2}=\left\|\chi_{0} u\right\|_{H^{\prime}(\Omega)}^{2}+\sum_{l>0}\left\|\chi_{l} u\right\|_{H_{\tan }^{1}(\Omega)}^{2}, \\
\left\|\chi_{l} u\right\|_{H_{\tan }^{1}(\Omega)}^{2}=\left\|u^{l}\right\|_{L^{2}\left(B_{-}\right)}^{2}+\left\|x_{1} D_{1} u^{l}\right\|_{L^{2}\left(B_{-}\right)}^{2}+\sum_{k \geqslant 2}\left\|D_{k} u^{l}\right\|_{L^{2}\left(B_{-}\right)}^{2} .
\end{gathered}
$$


The norms arising from different choices of $\vartheta_{l}, \tau_{j}, \chi_{l}$ are equivalent. The space $H_{\tan }^{1}\left(\Omega^{c}\right)$ is defined in the same way with $\Omega$ replaced by $\Omega^{c}$, and $\Re_{-}$replaced by $\Re_{+}$ in (4.1) and (4.2). We say that $u \in H_{\tan }^{1}\left(\mathbf{R}^{n}\right)$ if $u \in L^{2}\left(\mathbf{R}^{n}\right),\left.u\right|_{\Omega} \in H_{\tan }^{1}(\Omega),\left.u\right|_{\Omega^{c}} \in$ $H_{\tan }^{1}\left(\Omega^{c}\right)$. The norm is

$$
\|u\|_{H_{\tan }^{1}\left(\mathbf{R}^{n}\right)}^{2}=\|u\|_{H_{\tan }^{1}(\Omega)}^{2}+\|u\|_{H_{\tan }^{1}\left(\Omega^{c}\right)}^{2} .
$$

For the problem of vanishing viscosity we will need higher order tangential spaces (actually only $H_{\tan }^{2}$ ). These are defined as follows. For any integer $j \geqslant 0$,

$$
\begin{aligned}
& H_{\tan }^{j}(\Omega)=\left\{u \in L^{2}(\Omega): \text { for any } j\right. \text { tangential vector } \\
& \qquad \begin{array}{l}
\text { fields } \Lambda^{1}, \ldots, \Lambda^{j}, \Lambda^{1} \Lambda^{2} \cdots \Lambda^{j} u \in L_{\tan }^{2}(\Omega), \\
\text { and } \left.u \in H^{j}(\{x \in \Omega: \operatorname{dist}(x, \partial \Omega)>1\})\right\} .
\end{array}
\end{aligned}
$$

For $j=2$, the norm is

$$
\begin{aligned}
\|u\|_{H^{2}(\Omega)}^{2} & =\left\|\chi_{0} u\right\|_{H^{2}(\Omega)}+\sum_{l>0}\left\|\chi_{l} u\right\|_{H_{\mathrm{tan}}^{2}(\Omega)}^{2}, \\
\left\|\chi_{l} u\right\|_{H_{\mathrm{tan}}^{2}(\Omega)}^{2} & =\left\|x_{1} D_{1} u^{l}\right\|_{H_{\mathrm{tan}}^{1}\left(\mathscr{B}_{-}\right)}^{2}+\sum_{k=2}^{n}\left\|D_{k} u^{l}\right\|_{H_{\mathrm{tan}}^{1}\left(\mathscr{B}_{-}\right)}^{2}+\left\|u^{l}\right\|_{L^{2}\left(\oiint_{-}\right)}^{2} .
\end{aligned}
$$

THEOREM 5. There is $a \delta_{1} \geqslant \delta_{0}$ and $c>0$ so that if $\delta>\delta_{1}, \lambda \geqslant 0$ and $\varepsilon>0$ then:

(1) If $f \in H_{\tan }^{1}(\Omega)$ and $u_{\varepsilon}$ is the solution of (2.7) which arises in Theorem 1, then,

$$
\varepsilon \delta\left\|\nabla u_{\varepsilon}\right\|_{H_{\tan }^{1}(\Omega)}^{2}+\delta^{2}\left\|u_{\varepsilon}\right\|_{H_{\tan }^{1}(\Omega)}^{2} \leqslant c\|f\|_{H_{\tan }^{1}(\Omega)}^{2},
$$

and if $f \in H^{2}(\Omega)$, then $u_{\varepsilon} \in H^{3}(\Omega)$ and

$$
\varepsilon \delta\left\|\nabla u_{\varepsilon}\right\|_{H_{\mathrm{tan}}^{2}(\Omega)}^{2}+\delta^{2}\left\|u_{\varepsilon}\right\|_{H_{\mathrm{tan}}^{2}(\Omega)}^{2} \leqslant c\|f\|_{H_{\mathrm{tan}}^{2}(\Omega)}^{2} .
$$

(2) If $f \in H_{\tan }^{1}\left(\mathbf{R}^{n}\right)$ and $u_{\lambda}$ is the solution of (2.9) which arises in Theorem 2, then $u_{\lambda} \in H_{\mathrm{tan}}^{1}\left(\mathbf{R}^{n}\right)$ and

$$
\delta^{2}\left\|u_{\lambda}\right\|_{H_{\tan }^{1}(\Omega)}^{2}+\lambda \delta\left\|u_{\lambda}\right\|_{H_{\tan }^{1}\left(\Omega^{c}\right)}^{2} \leqslant c\|f\|_{H_{\tan }^{1}\left(\mathbf{R}^{n}\right)}^{2} .
$$

(3) If $f \in L^{2}\left([0, T]: H_{\tan }^{1}\left(\mathbf{R}^{n}\right)\right) \cap H^{1}\left([0, T]: L^{2}\left(\mathbf{R}^{n}\right)\right), g \in H_{\tan }^{1}\left(\mathbf{R}^{n}\right),\left.g\right|_{\Omega^{c}}=0$, and $u_{\lambda}$ is the solution of (2.12), (2.14) which arises in Theorem 3 , then

$$
u_{\lambda} \in C\left([0, T]: H_{\tan }^{1}\left(\mathbf{R}^{n}\right)\right) \cap C^{1}\left([0, T]: L^{2}\left(\mathbf{R}^{n}\right)\right)
$$

and

$$
\begin{aligned}
\sup _{0 \leqslant t \leqslant T}\left(\left\|u_{\lambda}(t)\right\|_{H_{\tan }^{1}\left(\mathbf{R}^{n}\right)}^{2}\right. & \left.+\left\|\frac{\partial u_{\lambda}}{\partial t}(t)\right\|_{L^{2}\left(\mathbf{R}^{n}\right)}^{2}\right) \\
& +\lambda\left(\int_{0}^{T}\left\|u_{\lambda}(t)\right\|_{H_{\tan }^{1}\left(\Omega^{c}\right)}^{2}+\left\|\frac{\partial u_{\lambda}}{\partial t}(t)\right\|_{L^{2}\left(\Omega^{c}\right)}^{2} d t\right) \\
\leqslant & c\left(\|g\|_{H_{\tan }^{1}(\Omega)}^{2}+\int_{0}^{T}\|f(t)\|_{H_{\tan }^{1}\left(\mathbf{R}^{n-1}\right)}^{2}+\left\|\frac{\partial f}{\partial t}(t)\right\|_{L^{2}\left(\mathbf{R}^{n}\right)}^{2} d t\right) .
\end{aligned}
$$


(4) If $f$ and $g$ are as in (3) with $\left.g\right|_{\Omega^{c}}=0$ and $u_{\lambda}$ is the solution of (2.13), (2.14) which arises in Theorem 4, then $u_{\lambda} \in C\left([0, T]: H_{\tan }^{1}\left(\mathbf{R}^{n}\right)\right) \cap C^{1}\left([0, T]: L^{2}\left(\mathbf{R}^{n}\right)\right)$ and

$$
\begin{gathered}
\sup _{0 \leqslant t \leqslant T}\left(\left\|u_{\lambda}(t)\right\|_{H_{\mathrm{tan}}^{1}\left(\mathbf{R}^{n}\right)}^{2}+\lambda\left\|u_{\lambda}(t)\right\|_{H_{\mathrm{tan}}^{1}\left(\Omega^{c}\right)}^{2}+\left\|\frac{\partial u_{\lambda}}{\partial t}(t)\right\|_{L^{2}\left(\mathbf{R}^{n}\right)}^{2}\right. \\
\left.+\lambda\left\|u_{\lambda}(t)\right\|_{H_{\mathrm{tan}}^{1}\left(\Omega^{c}\right)}^{2}+\lambda\left\|\frac{\partial u_{\lambda}}{\partial t}(t)\right\|_{L^{2}\left(\Omega^{c}\right)}^{2}\right) \\
\leqslant c\left(\|g\|_{H_{\mathrm{tan}}^{1}(\Omega)}^{2}+\int_{0}^{T}\|f(t)\|_{H_{\mathrm{tan}}^{1}\left(\mathbf{R}^{n-1}\right)}^{2}+\left\|\frac{\partial f}{\partial t}(t)\right\|_{L^{2}\left(\mathbf{R}^{n}\right)}^{2} d t\right) .
\end{gathered}
$$

The proof of this theorem is rather technical and involves several ingredients. First one observes that it suffices to prove the estimates (1)-(4) for solutions which are known a priori to be quite smooth. The result then follows by applying the estimates to suitably mollified (regularized) functions. Since such arguments are standard we will omit them. To derive the a priori estimate there are two distinct ingredients. One must derive estimates for all derivatives away from the boundary of $\Omega$ and this can be done by standard energy estimates (Lemma 2). To derive the estimates near $\partial \Omega$, we localize the problem so that $\Omega$ becomes a half space bounded by the plane $x_{1}=0$. By a change of variables we then cast the differential equations in a convenient form (Lemma 3 ). Notice that the reductions are different from those used in [1] or in $\S 3$.

The first step is the proof of the estimates away from $\partial \Omega$.

LEMMA 2 (A PRIORI INTERIOR ESTIMATES). For any $\psi \in \mathscr{D}\left(\mathbf{R}^{n}\right)$ with $\psi=1$ on a neighborhood of $\partial \Omega$ and $\varphi=1-\psi$, there are constants $\delta_{2} \geqslant \delta_{0}$ and $c>0$ so that if $\delta>\delta_{2}, \lambda \geqslant 0, \varepsilon>0$ :

(1) If $f$ and $u_{\varepsilon}$ are as in part (1) of Theorem 5 and $u_{\varepsilon} \in C^{\infty}(\bar{\Omega})$, then

$$
\begin{aligned}
\varepsilon \delta\left\|\left(\varphi u_{\varepsilon}\right)\right\|_{H^{2}(\Omega)}^{2} & +\delta^{2}\left\|\varphi u_{\varepsilon}\right\|_{H^{1}(\Omega)}^{2} \\
& \leqslant c\left(\|f\|_{H_{\mathrm{tan}}^{1}(\Omega)}^{2}+\varepsilon\left\|u_{\varepsilon}\right\|_{H_{\mathrm{tan}}^{1}(\Omega)}^{2}+\varepsilon\left\|u_{\varepsilon}\right\|_{H_{\mathrm{tan}}^{2}(\Omega)}^{2}+\left\|u_{\varepsilon}\right\|_{H_{\mathrm{tan}}^{1}(\Omega)}^{2}\right)
\end{aligned}
$$

and

$$
\varepsilon \delta\left\|\varphi u_{\varepsilon}\right\|_{H^{3}(\Omega)}^{2}+\delta^{2}\left\|\varphi u_{\varepsilon}\right\|_{H^{2}(\Omega)}^{2} \leqslant c\left(\|f\|_{H_{\mathrm{tan}}^{2}(\Omega)}^{2}+\varepsilon\left\|u_{\varepsilon}\right\|_{H_{\mathrm{tan}}^{3}(\Omega)}^{2}+\left\|u_{\varepsilon}\right\|_{H_{\mathrm{tan}}^{2}(\Omega)}^{2}\right)
$$

(2) If $f$ and $u_{\lambda}$ are as in part (2) of Theorem 5 and $u_{\lambda} \in C_{(0)}^{\infty}(\bar{\Omega}) \cap C_{(0)}^{\infty}\left(\bar{\Omega}^{c}\right)$, then

$$
\delta^{2}\left\|\varphi u_{\lambda}\right\|_{H^{\prime}(\Omega)}^{2}+\lambda \delta\left\|\varphi u_{\lambda}\right\|_{H^{\prime}(\Omega)}^{2} \leqslant c\left(\|f\|_{H_{\tan }^{1}\left(\mathbf{R}^{n}\right)}^{2}+\left\|u_{\lambda}\right\|_{H_{\tan }^{1}\left(\mathbf{R}^{n}\right)}^{2}\right)
$$

(3) If $f, g$, and $u_{\lambda}$ are as in part (3) of Theorem 5 and $u_{\lambda} \in C_{(0)}^{\infty}([0, T] \times \bar{\Omega}) \cap$ $C_{(0)}^{\infty}\left([0, T] \times \bar{\Omega}^{c}\right)$, then 


$$
\begin{aligned}
& \sup _{0 \leqslant t \leqslant T}\left(\left\|\varphi u_{\lambda}(t)\right\|_{H^{1}\left(\mathbf{R}^{n}\right)}^{2}+\left\|\varphi \partial_{t} u_{\lambda}(t)\right\|_{L^{2}\left(\mathbf{R}^{n}\right)}^{2}+\lambda\left\|\varphi u_{\lambda}(t)\right\|_{H^{1}\left([0, t] \times \Omega^{c}\right)}^{2}\right) \\
& \leqslant c\left(\|g\|_{H_{\tan }^{1}(\Omega)}^{2}+\int_{0}^{T}\|f(t)\|_{H_{\tan }^{1}(\Omega)}^{2}+\left\|\frac{\partial f}{\partial t}(t)\right\|_{L^{2}\left(\mathbf{R}^{n}\right)}^{2}\right. \\
&\left.+\left\|u_{\lambda}(t)\right\|_{H_{\tan \left(\mathbf{R}^{n}\right)}^{2}}^{2}+\left\|\partial_{t} u_{\lambda}(t)\right\|_{L^{2}\left(\mathbf{R}^{n}\right)}^{2} d t\right)
\end{aligned}
$$

(4) If $f, g$ and $u_{\lambda}$ are as in part (4) of Theorem 5 and $u_{\lambda} \in C_{(0)}^{\infty}([0, T] \times \bar{\Omega}) \cap$ $C_{(0)}^{\infty}\left([0, T] \times \bar{\Omega}^{c}\right)$, then

$$
\begin{gathered}
\sup _{0 \leqslant t \leqslant T}\left(\left\|\varphi u_{\lambda}(t)\right\|_{H^{1}(\Omega)}^{2}+\lambda\left\|\varphi u_{\lambda}(t)\right\|_{H^{1}\left(\Omega^{c}\right)}^{2}+\left\|\varphi \partial_{t} u_{\lambda}(t)\right\|_{L^{2}(\Omega)}^{2}+\lambda\left\|\varphi \partial_{t} u_{\lambda}(t)\right\|_{L^{2}\left(\Omega^{c}\right)}^{2}\right) \\
\leqslant c\left(\|g\|_{H_{\mathrm{tan}}^{1}(\Omega)}^{2}+\int_{0}^{T}\left(\|f(t)\|_{H_{\tan }^{1}\left(\mathbf{R}^{n}\right)}^{2}+\left\|\frac{\partial f}{\partial t}(t)\right\|_{L^{2}\left(\mathbf{R}^{n}\right)}^{2}\right.\right. \\
+\left\|u_{\lambda}(t)\right\|_{H_{\mathrm{tan}}^{1}\left(\mathbf{R}^{n}\right)}^{2}+\left\|\partial_{t} u_{\lambda}(t)\right\|_{L^{2}\left(\mathbf{R}^{n}\right)}^{2} \\
\left.\left.+\lambda\left\|u_{\lambda}(t)\right\|_{H_{\tan \left(\Omega^{c}\right)}^{2}}^{2}+\lambda\left\|\partial_{t} u_{\lambda}(t)\right\|_{L^{2}\left(\Omega^{c}\right)}^{2}\right) d t\right) .
\end{gathered}
$$

Proof of Lemma 2. These are all straightforward applications of the energy method. We will sketch the proofs in cases (1) and (4); the others are similar. Consider $u_{\varepsilon}$ as in part (1). We have for any $i$,

$$
\left(-\varepsilon \sum \frac{\partial}{\partial x_{i}} E_{i j} \frac{\partial}{\partial x_{j}}+\delta+L\right) \frac{\partial}{\partial x_{i}}\left(\varphi u_{\varepsilon}\right)=\frac{\partial \varphi f}{\partial x_{i}}+M_{\varepsilon}\left(u_{\varepsilon}\right)
$$

where $M_{\varepsilon}$ is a differential operator of order two whose terms of order two have coefficients $O(\varepsilon)$ and all of whose coefficients vanish in a neighborhood of $\partial \Omega$. Multiply this equation by $\partial \varphi u_{\varepsilon} / \partial x_{i}$ and integrate by parts to obtain the estimate

$$
\begin{aligned}
\varepsilon\left\|\nabla\left(\frac{\partial \varphi u_{\varepsilon}}{\partial x_{i}}\right)\right\|_{L^{2}(\Omega)}^{2} & +\delta\left\|\frac{\partial \varphi u_{\varepsilon}}{\partial x_{i}}\right\|_{L^{2}(\Omega)}^{2} \\
& \leqslant c\left\|\frac{\partial \varphi u_{\varepsilon}}{\partial x_{i}}\right\|_{L^{2}(\Omega)}\left(\|f\|_{H_{\mathrm{tan}}^{1}(\Omega)}+\varepsilon\left\|u_{\varepsilon}\right\|_{H_{\mathrm{tan}}^{2}(\Omega)}+\left\|u_{\varepsilon}\right\|_{H_{\mathrm{tan}}^{\mathrm{an}}(\Omega)}\right) .
\end{aligned}
$$

Summing over $i$ yields the first estimate of part (1). The second estimate is derived in the same fashion.

Next we pass to part (4). In this case, if $v=\partial\left(\varphi u_{\lambda}\right)$ with $\partial=\partial / \partial x_{i}, i=1, \ldots, n$, or $\partial=\partial_{t}$, then

$$
\left(L+\lambda \tilde{A}_{0}(\partial / \partial t)\right) v=f_{\lambda}
$$

where by explicit calculation of $f_{\lambda}$ one finds

$$
\begin{aligned}
\left\|f_{\lambda}(t)\right\|_{L^{2}\left(\mathbf{R}^{n}\right)} \leqslant c( & \lambda\left\|u_{\lambda}(t)\right\|_{H_{\tan }^{1}\left(\Omega^{c}\right)}+\left\|u_{\lambda}(t)\right\|_{H_{\tan }^{1}(\Omega)} \\
& \left.+\left\|\varphi \partial_{t} f(t)\right\|_{L^{2}\left(\mathbf{R}^{n}\right)}+\|\varphi f(t)\|_{H^{1}\left(\mathbf{R}^{n}\right)}+\|f(t)\|_{L^{2}\left(\mathbf{R}^{n}\right)}\right) .
\end{aligned}
$$


The standard energy method for (4.3) (note there is no problem with boundaries since $v=0$ near $\partial \Omega$ ) yields the estimate of part (4). This completes the proof of Lemma 2.

Next we describe the localizations required in the proof of the tangential estimates near $\partial \Omega$.

LEMMa 3. Assume that the entries of the right-hand side of

$$
a_{1}(x)=U(x)\left(\begin{array}{ccc}
a_{+}(x), & 0, & 0 \\
0, & a_{-}(x), & 0 \\
0, & 0, & 0
\end{array}\right) U^{*}(x)
$$

belong to $C^{3}\left(\mathbf{R}^{n}\right), a_{1}(x)$ is a $k \times k$ hermitian matrix, $U(x)$ is unitary, $a_{ \pm}(x)$ is a strictly positive (negative) diagonal matrix. Let $a_{k}(x)(2 \leqslant k \leqslant n)$ be $(n-1), C^{3}\left(\mathbf{R}^{n}\right)$, $k \times k$, hermitian matrix-valued functions. Let $e_{i j}(x)(1 \leqslant i, j \leqslant n)$ be $n^{2}, C^{2}\left(\mathbf{R}^{n}\right)$, $k \times k$, hermitian matrix-valued functions, $b_{k}, k=1, \ldots, n$, be $n C^{2}$ matrix-valued functions. Assume that one has

$$
\sum\left\langle e_{i j} \xi_{i}, \xi_{j}\right\rangle \geqslant \alpha \sum_{k=1}^{n}\left|\xi_{k}\right|^{2} \quad \forall\left(\xi_{1}, \xi_{2}, \ldots, \xi_{n}\right) \in\left(\mathbf{R}^{m}\right)^{n} \quad(\alpha>0) .
$$

Assume that $p(x)$ is a hermitian matrix-valued function with the properties:

$p(x)=0$ if $x \in \mathbf{R}_{--}^{n}$;

$\left.p(x)\right|_{\mathbf{R}_{+}^{n}}$ belongs to $C^{2} \overline{\left(\mathbf{R}_{+}^{n}\right)}$ and is strictly positive.

Then for any $\varepsilon \geqslant 0, \delta>0, \lambda>0$, the equation

$$
-\varepsilon\left[D_{i}\left(e_{i j} D_{j} v\right)+b_{k} D_{k} v\right]+a_{k} D_{k} v+\delta v+\lambda p v=f
$$

can be reduced to an equation of the same type but with "leading" coefficient $a_{1}$ independent of $x$. More precisely, there exists a matrix $V\left(V \in C^{3}\left(\mathbf{R}^{n}\right), V^{-1} \in C^{3}\left(\mathbf{R}^{n}\right)\right)$ such that $\tilde{v}=V^{-1} v$ is a solution of the equation

$$
-\varepsilon D_{i}\left(\tilde{e}_{i j} D_{j} \tilde{v}\right)+\varepsilon \tilde{b}_{k} D_{k} \tilde{v}+\tilde{a}_{k} D_{k} \tilde{v}+\left(\delta \tilde{q}+r_{1}+\varepsilon r_{2}\right) \tilde{v}+\lambda \tilde{p} \tilde{v}=V^{*} f
$$

which has the following properties:

(1) the matrices $\tilde{a}_{i}$ are hermitian symmetric;

(2) the operator $D_{j}\left(\tilde{e}_{i j} D_{i}\right)$ is elliptic in the sense of (4.4);

(3) the matrix $\tilde{q}$ is strictly positive;

(4) the matrix $\tilde{p}$ is zero on $\mathbf{R}_{-}^{n}$ and is $C^{2} \overline{\left(\mathbf{R}_{+}^{n}\right)}$ and strictly positive in $\overline{\mathbf{R}_{+}^{n}}$;

(5) the matrices $\tilde{e}_{i j}, \tilde{b}_{k}, \tilde{a}_{k}, \tilde{q}, r_{i}$ are smooth (belong to $C^{2}\left(\mathbf{R}^{n}\right)$ ) and $\tilde{a}_{1}$ is independent of $x$.

Proof. We introduce the matrix

$$
V(x)=U(x)\left[\begin{array}{ccc}
\sqrt{a_{+}(x)}, & 0, & 0 \\
0, & \sqrt{-a_{-}(x)}, & 0 \\
0, & 0, & 1
\end{array}\right] ;
$$


then we have

$$
\tilde{a}_{1}(x)=V^{*}(x) a_{1}(x) V(x)=\left(\begin{array}{ccc}
I, & 0, & 0 \\
0, & -I, & 0 \\
0, & 0, & 0
\end{array}\right)
$$

Now we put $v=V \tilde{v}$ and we multiply both sides of (4.5) by $V^{*}$. The system takes the form (4.6). The properties (1)-(5) are readily verified.

The next result gives the basic a priori estimates for the tangential derivatives near $\partial \Omega$. The proof is rather technical; the main ingredients are: (1) the localization of Lemma 3, and (2) the observation that the localized operators nearly commute with tangential derivatives in the sense that the commutator involves only first order derivatives in the tangential directions.

LEMMa 4. Assume that the coefficients $e_{i j}, a_{k}, b_{k}, p$ satisfy the hypotheses of Lemma 3. In addition suppose that $a_{0} \in C^{2}\left([0, T] \times \overline{\mathscr{B}}: \operatorname{Hom}\left(C^{k}\right)\right)$ is hermitian and strictly positive and that $\hat{a}_{0}$ is a $C^{2}$ hermitian strictly positive matrix on $[0, T] \times \overline{\mathscr{B}}_{+}$, and that $\hat{a}_{0}=0$ for $x \in \Re_{-}$. Then there are constants $\delta_{3} \geqslant \delta_{0}$ and $c>0$ so that for all $\lambda \geqslant 0$, $\delta \geqslant \delta_{3}$ and $0<\varepsilon$ :

(1) If $v \in H^{3}\left(\mathbf{R}_{-}^{n}\right) \cap \stackrel{\circ}{H}^{1}\left(\mathbf{R}_{-}^{n}\right)$, supp $v \in \Re_{-}$satisfies

$$
-\varepsilon D_{j} e_{i j} D_{i} v+a_{k} D_{k} v+\delta v=g \text {, }
$$

then for $i=1$ or $i=2$,

$$
\varepsilon \delta\|\nabla v\|_{H_{\mathrm{tan}}^{i}\left(\mathbf{R}_{-}^{n}\right)}^{2}+\delta^{2}\|v\|_{H_{\mathrm{tan}}^{i}\left(\mathbf{R}_{-}^{n}\right)}^{2} \leqslant\|g\|_{H_{\mathrm{tan}}^{i}\left(\mathbf{R}_{-}^{n}\right)}^{2} .
$$

(2) If $v \in C^{\infty} \overline{\left(\mathbf{R}_{+}^{n}\right)} \cap C^{\infty} \overline{\left(\mathbf{R}_{-}^{n}\right)} \cap C\left(\mathbf{R}^{n}\right)$, supp $v \in \mathscr{B}$ satisfies

$$
a_{k} D_{k} v+\lambda p v+\delta v=g \text {, }
$$

then

$$
\delta^{2}\|v\|_{H_{\tan }^{1}\left(\mathbf{R}^{n}\right)}^{2}+\lambda \delta\|v\|_{H_{\tan }^{1}\left(\mathscr{B}_{+}\right)}^{2} \leqslant c\|g\|_{H_{\tan }^{1}\left(\mathbf{R}^{n}\right)}^{2} .
$$

(3) If $v \in C^{\infty}\left([0, T] \times \overline{\mathbf{R}_{-}^{n}}\right) \cap C^{\infty}\left([0, T] \times \overline{\mathbf{R}_{+}^{n}}\right) \cap C\left([0, T] \times \mathbf{R}^{n}\right)$, supp $v \subset$ $[0, T] \times \Re$ satisfies

$$
a_{0}(\partial v / \partial t)+a_{k} D_{k} v_{k}+\lambda p v=g
$$

then if

$$
\Psi(t)=\|v(t)\|_{H_{\tan }^{1}\left(\mathbf{R}^{n}\right)}^{2}+\|\partial v(t) / \partial t\|_{L^{2}\left(\mathbf{R}^{n}\right)}^{2}
$$

and $0 \leqslant t \leqslant T$,

$$
\begin{aligned}
\Psi(t)+\lambda \int_{0}^{t} \| v(s) & \left\|_{H_{\tan \left(\mathbf{R}_{+}^{n}\right)}^{2}}^{t}+\right\| \frac{\partial v(s)}{\partial t} \|_{L^{2}\left(\mathbf{R}_{+}^{n}\right)}^{2} d s \\
& \leqslant c \Psi(0)+c \int_{0}^{t}\|g(s)\|_{H_{\mathrm{tan}}^{1}(\Omega)}^{2}+\left\|\frac{\partial g}{\partial s}(s)\right\|_{L^{2}\left(\mathbf{R}^{n}\right)}^{2} d s .
\end{aligned}
$$


(4) If $v \in C^{\infty}\left([0, T] \times \overline{\mathbf{R}_{-}^{n}}\right) \cap C^{\infty}\left([0, T] \times \overline{\mathbf{R}_{+}^{n}}\right), a_{1} v \in C\left([0, T] \times \mathbf{R}^{n}\right)$, supp $v \subset$ $[0, T] \times \Re$ satisfies

$$
\left(a_{0}+\lambda \hat{a}_{0}\right)(\partial v / \partial t)+a_{k} D_{k} v=g
$$

then if

$$
\begin{aligned}
\Phi(t)= & \|v(t)\|_{H_{\mathrm{tan}}^{1}\left(\mathbf{R}_{-}^{n}\right)}^{2}+\|\partial v(t) / \partial t\|_{L^{2}\left(\mathbf{R}_{-}^{n}\right)}^{2} \\
& +\lambda\|v(t)\|_{H_{\mathrm{tan}}^{1}\left(\mathbf{R}_{+}^{n}\right)}^{2}+\lambda\|\partial v(t) / \partial t\|_{L^{2}\left(\mathbf{R}_{+}^{n}\right)}^{2}
\end{aligned}
$$

and $0 \leqslant t \leqslant T$,

$$
\Phi(t) \leqslant c \Phi(0)+c \int_{0}^{t}\|g(s)\|_{H_{\tan \left(\mathbf{R}^{n}\right)}^{1}}^{2}+\left\|\frac{\partial g}{\partial t}(s)\right\|_{L^{2}\left(\mathbf{R}^{n}\right)}^{2} d s .
$$

Proof. We prove (1) and (4). The proofs of (2) and (3) follow the same lines and are somewhat simpler.

Let $v$ be the solution of (4.9). We apply Lemma 3 to cast equation (4.9) in the form

$$
{ }_{-\varepsilon} D_{i} \tilde{e}_{i j} D_{j} \tilde{v}+\tilde{a}_{1} D_{1} \tilde{v}+\sum_{k=2}^{n} \tilde{a}_{k} D_{k} \tilde{v}+\delta \tilde{q} v=\tilde{g}
$$

where $\tilde{a}_{1}$ is independent of $x$ and

$$
\|\tilde{g}\|_{H_{\mathrm{tan}}^{\mathrm{l}}} \leqslant c\left(\|g\|_{H_{\mathrm{tan}}^{\mathrm{l}}}+\|v\|_{H_{\mathrm{tan}}^{\mathrm{l}}}+\varepsilon\|\nabla v\|_{H_{\mathrm{lan}}^{\mathrm{lan}}}\right)
$$

where here and in all estimates up to (4.25) the norms are over $\mathbf{R}_{-}^{n}$. Let $M_{\varepsilon}$ be the differential operator $-\varepsilon D_{i} \tilde{e}_{i j} D_{j}+\tilde{a}_{k} D_{k}+\delta \tilde{q}$ so that (4.18) takes the form $M_{\varepsilon} \tilde{v}=\tilde{g}$. Take the real part of the scalar product of this equation with $\tilde{v}$ and integrate over $\mathbf{R}_{-}^{n}$ to obtain for $\delta$ large

$$
\varepsilon\|\nabla \tilde{v}\|_{L^{2}}^{2}+\delta\|\tilde{v}\|_{L^{2}}^{2} \leqslant\|\tilde{g}\|_{L^{2}}\|\tilde{v}\|_{L^{2}} .
$$

Using the analogue of (4.19) with $H_{\mathrm{tan}}^{1}$ replaced by $L^{2}$ we find that the right-hand side of (4.20) is dominated by $(\varepsilon / 2)\|\nabla \tilde{v}\|_{L^{2}}^{2}+c\|\tilde{v}\|_{L^{2}}^{2}+c\|g\|_{L^{2}}^{2}$. This yields the basic estimate

$$
\varepsilon \delta\|\nabla \tilde{v}\|_{L^{2}}^{2}+\delta^{2}\|\tilde{v}\|_{L^{2}}^{2} \leqslant c\|\tilde{g}\|_{L^{2}}^{2} .
$$

To estimate $\|\tilde{v}\|_{H_{\tan }^{1}}$ and therefore $\|v\|_{H_{\text {tan }}^{1}}$, we need estimates for $\left\|D_{r} \tilde{v}\right\|_{L^{2}}, 2 \leqslant r \leqslant n$, and $\left\|x_{1} D_{1} \tilde{v}\right\|_{L^{2}}$. Let $D$ represent either the operator $x_{1} D_{1}$ or $D_{r}$ for $r \geqslant 2$. Differentiating (4.18) yields

$$
M_{\varepsilon}(D \tilde{v})=D \tilde{g}+\left[M_{\varepsilon}, D\right] \tilde{v} .
$$

Taking the real part of the $L^{2}$ scalar product with $D \tilde{v}$ and using (4.21) to estimate $\varepsilon[\nabla, D] \tilde{v}$ yields for $\delta$ large

$$
\varepsilon\|D \nabla \tilde{v}\|_{L^{2}}^{2}+\delta\|D \tilde{v}\|_{L^{2}}^{2} \leqslant c\|\tilde{g}\|_{H_{\text {tan }}^{1}}^{2}+\operatorname{Re}\left(D \tilde{v},\left[M_{\varepsilon}, D\right] \tilde{v}\right) .
$$

We will show that for each $D$,

$$
\left|\operatorname{Re}\left(D \tilde{v},\left[M_{\varepsilon}, D\right] D \tilde{v}\right)\right| \leqslant c\left(\|\tilde{g}\|_{H_{\mathrm{tan}}^{1}}^{2}+\|\tilde{v}\|_{H_{\mathrm{tan}}^{1}}^{2}+\varepsilon\|\nabla \tilde{v}\|_{H_{\mathrm{tan}}^{1}}\|\tilde{v}\|_{H_{\mathrm{tan}}^{1}}\right) .
$$


Using this estimate in (4.23), summing over all $D$, and estimating

$$
\sum c \varepsilon\|\nabla \tilde{v}\|_{H_{\mathrm{tan}}^{1}}\|\tilde{v}\|_{H_{\mathrm{tan}}^{1}} \leqslant(\varepsilon / 2)\|\nabla \tilde{v}\|_{H_{\mathrm{tan}}^{1}}^{2}+c\|\tilde{v}\|_{H_{\mathrm{tan}}^{1}}^{2}
$$

yields the desired estimate.

To prove (4.24) we write

$$
M_{\varepsilon}=-\varepsilon D_{1} e_{11} D_{1}+a_{1} D_{1}+N_{\varepsilon}
$$

defining the operator $N_{\varepsilon}$. It is easy to see that

$$
\left\|\left[N_{\varepsilon}, D\right] \tilde{v}\right\|_{L^{2}} \leqslant c\left(\varepsilon\|\nabla \tilde{v}\|_{H_{\mathrm{tan}}^{1}}+\|\tilde{v}\|_{H_{\mathrm{tan}}^{1}}+\delta\|\tilde{v}\|_{L^{2}}\right) .
$$

This, together with (4.21), yields the desired estimate for $\left|\left(D \tilde{v},\left[N_{\varepsilon}, D\right] \tilde{v}\right)_{L^{2}}\right|$. The other commutator terms require more subtlety. Consider first the case $D=D_{r}$. Then

$$
\left[-\varepsilon D_{1} e_{11} D_{1}+a_{1} D_{1}, D_{r}\right]=-\varepsilon D_{1}\left(D_{r} e_{11}\right) D_{1} .
$$

Using the facts that $\tilde{v}$ and $D_{r} \tilde{v}$ vanish when $x_{1}=0$, two integrations by parts yield

$$
\left(D_{r} \tilde{v}, D_{1}\left(D_{r} e_{11}\right) D_{1} \tilde{v}\right)=\left(D_{1} \tilde{v}, D_{r}\left(D_{r} e_{11}\right) D_{1} \tilde{v}\right) \text {. }
$$

Now, by virtue of the symmetry of $D_{r} e_{11}$,

$$
\operatorname{Re}\left\langle D_{1} \tilde{v}, D_{r}\left(D_{r} e_{11}\right) D_{1} \tilde{v}\right\rangle=\frac{1}{2} D_{r}\left\langle D_{1} \tilde{v},\left(D_{r} e_{11}\right) D_{1} \tilde{v}\right\rangle-\left\langle D_{1} \tilde{v},\left(D_{r}^{2} e_{11}\right) D_{1} \tilde{v}\right\rangle .
$$

Since $v$ vanishes for $x_{r}$ large, the integral of the $D_{r}\langle\rangle$ term over $\mathbf{R}_{-}^{n}$ vanishes and one gets

$$
\left|\operatorname{Re}\left(D_{1} \tilde{v}, \varepsilon D_{r}\left(D_{r} e_{11}\right) D_{1} \tilde{v}\right)\right| \leqslant c \varepsilon\|\nabla \tilde{v}\|_{L^{2}}^{2} .
$$

Consider next the case $D=x_{1} D_{1}$. Here, the critical commutator is

$$
\left[-\varepsilon D_{1} e_{11} D_{1}+a_{1} D_{1}, x_{1} D_{1}\right]={ }_{-\varepsilon} D_{1} e_{11} D_{1}+a_{1} D_{1}-\varepsilon e_{11} D_{1}^{2}+\varepsilon x_{1}\left(D_{1}^{2} e_{11}\right) D_{1} \text {. }
$$

Using the equation $M_{\varepsilon} \tilde{v}=\tilde{g}$ we have

$$
\text { (commutator) } \tilde{v}=\tilde{g}-N_{\varepsilon} \tilde{v}+\varepsilon x_{1}\left(D_{1}^{2} e_{11}\right) D_{1} \tilde{v}-\varepsilon e_{11} D_{1}^{2} \tilde{v} .
$$

The scalar product of the first three terms on the right with $x_{1} D_{1} \tilde{v}$ is estimated using the Schwartz inequality and the estimate

$$
\left\|N_{\varepsilon} \tilde{v}\right\|_{L^{2}}+\left\|\varepsilon x_{1}\left(D_{1}^{2} e_{11}\right) D_{1} \tilde{v}\right\|_{L^{2}} \leqslant c\left(\varepsilon\|\nabla \tilde{v}\|_{H_{\mathrm{tan}}^{1}}+\|\tilde{v}\|_{H_{\mathrm{tan}}^{1}}+\delta\|\tilde{v}\|_{L^{2}}\right) .
$$

For the final term write $\Gamma \equiv-x_{1} e_{11}$, so we must estimate $\left|\operatorname{Re}\left(D_{1} \tilde{v}, \varepsilon \Gamma D_{1}^{2} \tilde{v}\right)\right|$. The symmetry of $\Gamma$ implies that

$$
\operatorname{Re}\left\langle D_{1} \tilde{v}, \Gamma D_{1}^{2} \tilde{v}\right\rangle=\frac{1}{2} D_{1}\left\langle D_{1} \tilde{v}, \Gamma D_{1} \tilde{v}\right\rangle-\left\langle D_{1} \tilde{v},\left(D_{1} \Gamma\right) D_{1} \tilde{v}\right\rangle
$$

Integrating over $\mathbf{R}_{-}^{n}$ the integral of $D_{1}\left\langle>\right.$ vanishes since $\Gamma=0$ when $x_{1}=0$. Thus

$$
\left|\operatorname{Re}\left(D_{1} \tilde{v}, \varepsilon \Gamma D_{1}^{2} \tilde{v}\right)\right| \leqslant c \varepsilon\|\nabla \tilde{v}\|_{L^{2}}^{2} \leqslant\|\tilde{g}\|_{L^{2}}^{2} .
$$

The proof of (4.24) and therefore (4.10) with $i=1$ is complete.

To prove (4.10) for $i=2$ one proceeds as before. With $D^{\prime}$ a second tangential derivative, one differentiates (4.22) to obtain

$$
M\left(D^{\prime} D \tilde{v}\right)=D^{\prime} D g+D^{\prime}[M \varepsilon, D] \tilde{v}+\left[M_{\varepsilon}, D^{\prime}\right] D \tilde{v} .
$$

Next one takes the real part of the $L^{2}$ scalar product with $D^{\prime} D v$. The crucial estimate analogous to (4.24) is

$$
\left|\operatorname{Re}\left(D^{\prime} D \tilde{v}, D^{\prime}\left[M_{\varepsilon}, D\right] \tilde{v}\right)\right| \leqslant c\left(\|\tilde{g}\|_{H_{\text {tan }}^{2}}^{2}+\|\tilde{v}\|_{H_{\text {tan }}^{2}}^{2}+\varepsilon\|\nabla v\|_{H_{\text {tan }}^{2}}\|\tilde{v}\|_{H_{\text {tan }}^{2}}\right)
$$


with a similar estimate for the term coming from the extreme right-hand element in (4.25). These estimates, which rest essentially on the symmetry of $e_{11}$ and $a_{1}$, are proved via integration by parts in a manner analogous to the proof of (4.24).

Next we prove part (4) of the lemma. Apply Lemma 3 to reduce (4.15) to a system

$$
\left(\tilde{a}_{0}+\lambda \tilde{a}_{0}\right) \frac{\partial v}{\partial t}+\sum_{1}^{n} \tilde{a}_{k} D_{k} \tilde{v}=\tilde{g}
$$

where $\tilde{a}_{1}$ is independent of $t, x$ and

$$
\begin{aligned}
&\|\tilde{g}(s)\|_{H_{\tan \left(\mathbf{R}^{n}\right)}^{1}}+\left\|\frac{\partial \tilde{g}}{\partial t}\right\|_{L^{2}\left(\mathbf{R}^{n}\right)} \\
& \leqslant c\left(\|g(s)\|_{H_{\tan \left(\mathbf{R}^{n}\right)}}+\left\|\frac{\partial g}{\partial t}(s)\right\|_{L^{2}\left(\mathbf{R}^{n}\right)}+\|\tilde{v}(s)\|_{H_{\tan \left(\mathbf{R}^{n}\right)}^{1}}\right. \\
&\left.+\left\|\frac{\partial \tilde{v}}{\partial t}\right\|_{L^{2}\left(\mathbf{R}^{n}\right)}+\lambda\|\tilde{v}\|_{H_{\tan }^{1}\left(\mathbf{R}_{+}^{n}\right)}+\lambda\left\|\frac{\partial \tilde{v}}{\partial t}\right\|_{L^{2}\left(\mathbf{R}_{+}^{n}\right)}\right) .
\end{aligned}
$$

Let $Q_{\lambda}$ be the differential operator $\left(\tilde{a}_{0}+\lambda \tilde{a}_{0}\right)(\partial / \partial t)+\sum_{1}^{n} a_{k} D_{k}$; then the basic energy estimate for $Q_{\lambda}$ is $\forall w \in C^{1}\left([0, T] \times \mathbf{R}_{-}^{n}\right) \cap C^{1}\left([0, T] \times \mathbf{R}_{+}^{n}\right)$ with $\tilde{a}_{1} w \in$ $C\left([0, T] \times \mathbf{R}^{n}\right)$ and supp $w \subset[0, T] \times \Re$.

$$
\eta(t) \leqslant c \eta(0)+c \int_{0}^{t} \eta(s)+\left|\left(w(s), Q_{\lambda} w(s)\right)\right| d s, \quad 0 \leqslant t \leqslant T,
$$

where

$$
\eta(s)=\|w(s)\|_{L^{2}\left(\mathbf{R}^{n}\right)}^{2}+\lambda\|w(s)\|_{L^{2}\left(\mathbf{R}_{+}^{n}\right)}^{2} .
$$

Applied to $w=\tilde{v}$ this gives for $0 \leqslant t \leqslant T$,

$$
\begin{aligned}
& \|\tilde{v}(t)\|_{L^{2}\left(\mathbf{R}^{n}\right)}^{2}+\lambda\|\tilde{v}(t)\|_{L^{2}\left(\mathbf{R}_{+}^{n}\right)}^{2} \\
& \quad \leqslant c\left(\|\tilde{v}(0)\|_{L^{2}\left(\mathbf{R}^{n}\right)}^{2}+\lambda\|\tilde{v}(0)\|_{L^{2}\left(\mathbf{R}_{+}^{n}\right)}^{2}+\int_{0}^{t}\|g(s)\|_{L^{2}\left(\mathbf{R}^{n}\right)}^{2} d s\right) .
\end{aligned}
$$

We want to estimate $D_{r} \tilde{v}$ for $2 \leqslant r \leqslant n, \partial \tilde{v} / \partial t$, and $x_{1} D_{1} \tilde{v}$. Differentiating equation (4.26) with respect to $x_{r}$ for $2 \leqslant r \leqslant n$ or with respect to $t$, one finds

$$
Q_{\lambda}(D \tilde{v})=D \tilde{g}+\left[Q_{\lambda}, D\right] \tilde{v}
$$

where $D=\partial / \partial t$ or $D=D_{r}, 2 \leqslant r \leqslant n$. Since $\tilde{a}_{1}$ is independent of $t, x$, the commutator satisfies $\left|\left(v(s),\left[Q_{\lambda}, D_{r}\right] v(s)\right)\right| \leqslant c \Phi(s)$, so by (4.27),

$$
\left\|Q_{\lambda}(D \tilde{v})(s)\right\|_{L^{2}\left(\mathbf{R}^{n}\right)}^{2} \leqslant c \Phi(s)+\|g(s)\|_{H_{\tan }^{1}\left(\mathbf{R}^{n}\right)}^{2}+\|\partial g(s) / \partial t\|_{L^{2}\left(\mathbf{R}^{n}\right)}^{2}
$$

where $\Phi$ is defined in part (4) of the lemma. Notice that $\tilde{a}_{1} D v=D \tilde{a}_{1} v$ is continuous on $[0, T] \times \mathbf{R}^{n}$ so we may apply the basic energy estimate (4.28) with $w=D \tilde{v}$. Similarly, differentiating equation (4.26) with respect to $x_{1}$ and multiplying by $x_{1}$ yields

$$
Q_{\lambda}\left(x_{1} D_{1} \tilde{v}\right)=x_{1} D_{1} \tilde{g}+\left[Q_{\lambda}, x_{1} D_{1}\right] \tilde{v} .
$$

Notice that even though $D_{1} \tilde{v}$ need not be continuous on $\mathbf{R}^{n}, x_{1} D_{1} \tilde{v}$ is and we may apply the basic energy estimate (4.28) with $w=x_{1} D_{1} \tilde{v}$. Toward this end observe that

$$
\left|\left(x_{1} D_{1} \tilde{v}(s),\left[Q_{\lambda}, x_{1} D_{1}\right] \tilde{v}(s)\right)\right| \leqslant c \Phi(s)+\left|\left(x_{1} D_{1} \tilde{v}(s), a_{1} D_{1} \tilde{v}(s)\right)\right| .
$$


However, the differential equation (4.26) shows that $\left|\left(x_{1} D_{1} \tilde{v}, a_{1} D_{1} \tilde{v}\right)\right| \leqslant c \Phi(s)+$ $\|\tilde{g}(s)\|_{L^{2}\left(\mathbf{R}^{n}\right)}^{2}$, so with (4.27) this yields

$$
\left|\left(x_{1} D_{1} \tilde{v}, Q_{\lambda}\left(x_{1} D_{1} \tilde{v}\right)\right)\right| \leqslant c \Phi(s)+c\|g(s)\|_{H_{\tan }^{1}\left(\mathbf{R}^{n}\right)}^{2} .
$$

Apply the basic identity (4.28) to $D_{r} \tilde{v}, 2 \leqslant r \leqslant n, \partial \tilde{v} / \partial t$, and $x_{1} D_{1} \tilde{v}$ using (4.30) and (4.31) to estimate the result of applying $Q_{\lambda}$ to these functions. Adding the resulting expressions to (4.29) yields (4.17) and thus completes the proof of Lemma 4.

END of Proof of Theorem 5. Using the estimates of Lemma 4 for the localizations $u_{\varepsilon}^{l}, u_{\lambda}^{l}, l \geqslant 1$, and the estimates of Lemma 2 for $\chi_{0} u_{\varepsilon}, \chi_{0} u_{\lambda}$ and summing yields the estimates of Theorem 5 . This process has only one hitch, that is, for the $u_{\varepsilon}^{l}$ the right-hand sides of the localized equations come from the right-hand side of the equations defining $u_{\lambda}$ or $u_{\varepsilon}$ and terms from the commutator of multiplication by $\chi^{l}$ with the differential operators entering the equations defining $u_{\lambda}, u_{\varepsilon}$. Both types of terms enter in the terms called $g$ in Lemma 4. The commutator terms are lower order. However, the commutators may have large coefficients. Nevertheless, they can be estimated by using the basic energy estimates (3.1)-(3.5). In deriving the $H_{\tan }^{2}(\Omega)$ estimate in the first part of Theorem 5 , the commutator terms are estimated by using the $H_{\text {tan }}^{1}$ estimates.

5. Strong convergence and the $H^{1 / 2-\eta}$ estimate. In this section we show how the tangential regularity of Theorem 5 can be used in the proofs of Theorems 1-4. First, using energy identities, the proofs of Theorems 2 and 4 are given. The proof of Theorem 3, which is similar to these, is omitted. Finally, we prove a uniform $H^{1 / 2-\eta}(\Omega)$ bound for the solutions $u_{\varepsilon}$ of problem (2.7), and the strong convergence follows easily. We call attention to the $H^{1 / 2-\eta}(\Omega)$ bound which is not an easy consequence of Theorem 5 and is of independent interest.

Proof of Theorem 2. Our notation is the same as in the statement of Theorem 2. Since the operators $\delta I+L+\lambda P$ are uniformly strictly accretive for $\delta>\delta_{0}, \lambda \geqslant 0$, it suffices to prove the assertion of the theorem for a single $\delta>\delta_{0}$. We will assume that $\delta>\delta_{1}$ where $\delta_{1}$ is as in Theorem 5. Since the inverse operators $(\delta I+L+\lambda P)^{-1}$ are uniformly bounded for $\lambda \geqslant 0$, it suffices to prove the convergence $u_{\lambda} \rightarrow u$ for $f$ in a dense set $\mathscr{F}$ in $L^{2}\left(\mathbf{R}^{n}\right)$. We take $\mathscr{F}=H_{\tan }^{1}\left(\mathbf{R}^{n}\right)$. Theorem 5 then yields a uniform estimate $\left\|u_{\lambda}\right\|_{H_{\tan }^{1}\left(\mathbf{R}^{n}\right)} \leqslant$ constant independent of $\lambda$.

The standard partial hypoellipticity argument shows that the map $\left.v \mapsto A_{\nu} v\right|_{\partial \Omega}$ is continuous from the Banach space, $\left\{v \in L^{2}(\Omega): L v \in L^{2}(\Omega)\right\}$ with norm $v \mapsto\|v\|_{L^{2}(\Omega)}+\|L v\|_{L^{2}(\Omega)}$, to $H^{-1 / 2}(\partial \Omega)$ (see [1]). Thus the weak convergence $u_{\lambda} \rightarrow u$ in $L^{2}(\Omega)$ and the differential equations $(\delta+L) u_{\lambda}=f$ in $\Omega$ imply that $\left.\left.A_{\nu} u_{\lambda}\right|_{\partial \Omega} \rightarrow A_{\nu} u\right|_{\partial \Omega}$ weakly in $H^{-1 / 2}(\partial \Omega)$. The same differential equations and the fact that $\left\{u_{\lambda}: \lambda \geqslant 0\right\}$ is bounded in $H_{\tan }^{1}(\Omega)$ imply that $\left\{A_{\nu} u_{\lambda}\right\}_{\lambda}$ is bounded in $H^{1}(\Gamma)$ where $\Gamma$ is any compact neighborhood of $\partial \Omega$ on which $\nu$ is smooth. Thus $\left\{\left.A_{\nu} u_{\lambda}\right|_{\partial \Omega}\right\}_{\lambda}$ is bounded in $H^{1 / 2}(\partial \Omega)$ and therefore precompact in $L^{2}(\partial \Omega)$. It follows that $\left.\left.A_{\nu} u_{\lambda}\right|_{\partial \Omega} \rightarrow A_{\nu} u\right|_{\partial \Omega}$ strongly in $L^{2}(\partial \Omega)$. 
We next show that, as $\lambda \rightarrow \infty$,

$$
\int_{\partial \Omega}\left\langle A_{\nu}\left(u_{\lambda}-u\right), u_{\lambda}-u\right\rangle d \sigma \rightarrow 0 .
$$

First to see that this integral is meaningful, let $K(x)$ for $x \in \partial \Omega$ be the hermitian operator on $\mathrm{C}^{k}$ defined by $\left.K(x)\right|_{\operatorname{null}\left(A_{\nu}\right)}=0,\left.K(x)\right|_{\operatorname{rg}\left(A_{\nu}\right)}=\left(\left.A_{\nu}\right|_{\operatorname{rg}\left(A_{\nu}\right)}\right)^{-1}$. Then $K A$ $=I$ on $\operatorname{rg} A_{\nu}$, so the integral is equal to

$$
\int_{\partial \Omega}\left\langle A_{\nu}\left(u_{\lambda}-u\right), K A_{\nu}\left(u_{\lambda}-u\right)\right\rangle d \sigma
$$

which makes sense since $A_{\nu}\left(u_{\lambda}-u\right) \in L^{2}(\partial \Omega)$. In addition, the convergence (5.1) follows from the fact that $A_{\nu}\left(u_{\lambda}-u\right) \rightarrow 0$ in $L^{2}(\partial \Omega)$.

The classical energy identity is

$$
\begin{aligned}
2\left(\delta-\delta_{0}\right)\left\|u_{\lambda}-u\right\|_{L^{2}(\Omega)}^{2} & \leqslant\left(\left[(\delta+L)+(\delta+L)^{*}\right]\left(u_{\lambda}-u\right), u_{\lambda}-u\right)_{L^{2}(\Omega)} \\
& =\operatorname{Re}\left((\delta+L)\left(u_{\lambda}-u\right),\left(u_{\lambda}-u\right)\right)_{L^{2}(\Omega)}+\int_{\partial \Omega}\left\langle A_{\nu}\left(u_{\lambda}-u\right), u_{\lambda}-u\right\rangle d \sigma .
\end{aligned}
$$

Thus, the convergence of $u_{\lambda}$ to $u$ in $L^{2}(\Omega)$ follows from (5.1) and the differential equations $(\delta+L)\left(u_{\lambda}-u\right)=0$ in $\Omega$.

Proof of TheOrem 4. As above, it suffices to prove that $u_{\lambda} \rightarrow u$ in $C\left([0, T]: L^{2}(\Omega)\right)$ for $f \in L^{2}\left([0, T]: H_{\tan }^{1}\left(\mathbf{R}^{n}\right)\right) \cap H^{1}\left([0, T]: L^{2}\left(\mathbf{R}^{n}\right)\right)$ and $g \in H_{\tan }^{1}\left(\mathbf{R}^{n}\right)$ with $\left.g\right|_{\Omega^{c}}=0$ since these are dense subsets of $L^{2}\left([0, T] \times \mathbf{R}^{n}\right)$ and $\left\{g \in L^{2}\left(\mathbf{R}^{n}\right)\right.$ : $\left.\left.g\right|_{\Omega^{c}}=0\right\}$, respectively. Then by Theorem $5,\left\{u_{\lambda}: \lambda \geqslant 0\right\}$ is bounded in $C\left([0, T]: H_{\tan }^{1}\left(\mathbf{R}^{n}\right)\right) \cap C^{1}\left([0, T]: L^{2}\left(\mathbf{R}^{n}\right)\right)$. As in the proof of Theorem $2, A_{\nu} u_{\lambda} \rightarrow A_{\nu} u$ weakly in $H^{-1 / 2}((0, T) \times \partial \Omega)$, and the tangential regularity implies that $\left\{A_{\nu} u_{\lambda}\right\}_{\lambda}$ is bounded in $H^{1}((0, T) \times \Gamma)$ where $\Gamma$ is a compact neighborhood of $\partial \Omega$. Thus $\left\{A_{\nu} u_{\lambda}\right\}$ is bounded in $H^{1 / 2}((0, T) \times \partial \Omega)$, hence precompact in $L^{2}((0, T) \times \partial \Omega)$ so $A_{\nu} u_{\lambda} \rightarrow$ $A_{\nu} u$ strongly in $L^{2}((0, T) \times \partial \Omega)$. For this problem, the energy identity in $[0, t] \times \Omega$ for $0 \leqslant t \leqslant T$ yields

$$
\begin{aligned}
\left\|\left(u_{\lambda}-u\right)(t)\right\|_{L^{2}(\Omega)}^{2} \leqslant & c \int_{0}^{t}\left\|\left(u_{\lambda}-u\right)(s)\right\|_{L^{2}(\Omega)}^{2} d s \\
& +\frac{1}{2} \iint_{[0, t] \times \partial \Omega}\left\langle A_{\nu}\left(u_{\lambda}-u\right), u_{\lambda}-u\right\rangle d t d \sigma \\
\leqslant & c \int_{0}^{t}\left\|\left(u_{\lambda}-u\right)(s)\right\|_{L^{2}(\Omega)}^{2} d s+c\left\|A_{\nu}\left(u_{\lambda}-u\right)\right\|_{L^{2}((0, t) \times \partial \Omega)}^{2} .
\end{aligned}
$$

Gronwall's inequality shows that

$$
\left\|u_{\lambda}-u\right\|_{C\left([0, T]: L^{2}(\Omega)\right)} \leqslant c\left\|A_{\nu}\left(u_{\lambda}-u\right)\right\|_{L^{2}((0, T) \times \partial \Omega)}
$$

and the proof is complete.

The strong convergence asserted in Theorem 1 is an immediate consequence of Theorem 6 which asserts the boundedness of $\left\{u_{\varepsilon}: 0<\varepsilon\right\}$ in $H^{1 / 2-\eta}(\Omega)$ for any $\eta>0$. This result is sharp in the sense that if $s>\frac{1}{2}$ then $u_{\varepsilon}$ will not, in general, be bounded in $H^{s}(\Omega)$. To see this, observe that if $u_{\varepsilon}$ were bounded in $H^{s}(\Omega)$ we would have $u_{\varepsilon} \rightarrow u$ in $H^{s}(\Omega)$ so the trace theorem yields $\left.\left.u_{\varepsilon}\right|_{\partial \Omega} \rightarrow u\right|_{\partial \Omega}$ in $H^{s-1 / 2}(\partial \Omega)$. Since 
$\left.u_{\varepsilon}\right|_{\partial \Omega}=0$ it follows that $u$ solves the boundary value problem $(L+\delta I) u=f$ in $\Omega$, $\left.u\right|_{\partial \Omega}=0$. Unless $A_{\nu}(x) \leqslant 0$ for all $x \in \partial \Omega$, there exist $f \in L^{2}(\Omega)$ for which this boundary value problem will have no solutions. Thus, except when $A_{\nu}$ is negative, we cannot expect to have $\left\{u_{\varepsilon}: \varepsilon \geqslant 0\right\}$ bounded in $H^{s}(\Omega)$ for any $s>\frac{1}{2}$. To see whether there is boundlessness in $H^{1 / 2}$ we consider the special case

$$
\begin{aligned}
\left(\varepsilon D_{1}^{2}+D_{1}\right) u_{\varepsilon} & =1, & & 0<x<1, \\
u_{\varepsilon} & =0, & & x=0,1,
\end{aligned}
$$

with explicit solution

$$
u_{\varepsilon}(x)=x-\left(1-e^{-x / \varepsilon}\right) /\left(1-e^{-1 / \varepsilon}\right) .
$$

In this case $u_{\varepsilon}$ is bounded in $H^{1 / 2}$ away from $x=0$ but is not bounded in $H^{1 / 2}(0, \sigma)$ for any $\sigma>0$.

The statement and proof of Theorem 6 are complicated by the fact that $\partial \Omega$ may be characteristic for $L$. In the noncharacteristic case one has $\left\|u_{\varepsilon}\right\|_{H^{1 / 2-\eta}(\Omega)} \leqslant$ $c\|f\|_{H_{\tan }^{1}(\Omega)}$. In the characteristic case the components of $u_{\varepsilon}$ in the nullspace of $(E \nu \cdot \nu)^{-1 / 2} A_{\nu}(E \nu \cdot \nu)^{-1 / 2}$ may be less smooth than the others. To compensate for this correspondingly more regularity of these components of $f$ is required in order to bound the $H^{1 / 2-\eta}$ norm of $u_{\varepsilon}$.

Theorem 6. Suppose $0<\eta$ and $P_{0}=\varphi \pi_{0} \in C_{0}^{1}\left(\bar{\Omega}: \operatorname{Hom}\left(C^{k}\right)\right)$ where $\varphi \in C^{\infty}\left(\mathbf{R}^{n}\right)$ is supported near $\partial \Omega, \varphi=1$ on a neighborhood of $\partial \Omega$, and $\pi_{0}$ is orthogonal projection on nullspace $(E \nu \cdot \nu)^{-1 / 2} A_{\nu}(E \nu \cdot \nu)^{-1 / 2}$. Then there are positive constants $\delta_{4} \geqslant \delta_{0}$ and $c$ so that if $0<\varepsilon, \delta \geqslant \delta_{4}$ and $u_{\varepsilon}$ is the solution of (2.7) then

(1) Regularity of $\left(I-P_{0}\right) u_{\varepsilon}$ : for all $f \in H_{\tan }^{1}(\Omega)$,

$$
\left\|\left(I-P_{0}\right) u_{\varepsilon}\right\|_{H^{1 / 2-\eta}(\Omega)}<c\|f\|_{H_{\tan }^{1}(\Omega)} \text {. }
$$

(2) Regularity of $P_{0} u_{\varepsilon}:$ for all $f \in H_{\tan }^{2}(\Omega)$ with $P_{0} f \in \stackrel{H}{ }^{1}(\Omega)$,

$$
\left\|P_{0} u_{\varepsilon}\right\|_{H^{1 / 2-\eta}(\Omega)}<c\left(\|f\|_{H_{\tan (\Omega)}^{2}}+\left\|P_{0} f\right\|_{H^{1}(\Omega)}\right) \text {. }
$$

REMARK. (1) The content of the assertions of Theorem 6 is independent of the choice of $P_{0}$, since if $P_{0}$ and $\tilde{P}_{0}$ are equal on $\partial \Omega$, then for any $g \in H_{\tan }^{1}(\Omega)$, $\left(P-\tilde{P}^{0}\right) g \in H^{1}(\Omega)$ and $\left\|\left(P_{0}-\tilde{P}_{0}\right) g\right\|_{H^{1}(\Omega)} \leqslant c\|g\|_{H_{\mathrm{tan}}^{1}(\Omega)}$. In particular the righthand side of (5.2) defines a norm and a corresponding Banach space which, up to equivalence of norms, does not depend on $P_{0}$.

Proof of Theorem 1 assuming Theorem 6. As in the proof of Theorems 2 and 4, we may assume that $\delta>\delta_{1}$ and $f \in H^{1}(\Omega) \cap H_{\tan }^{2}(\Omega)$. If $\Omega$ is bounded it follows from Theorem 6 that $\left\{u_{\varepsilon}: 0<\varepsilon\right\}$ is bounded in $H^{1 / 2-\eta}(\Omega)$ and therefore is precompact in $L_{2}(\Omega)$. Since $u_{\varepsilon} \rightarrow u$ weakly in $L^{2}(\Omega)$ it follows that $u_{\varepsilon} \rightarrow u$ strongly in $L^{2}(\Omega)$.

If $\Omega$ is not bounded, choose $\varphi \in C^{\infty}\left(\mathbf{R}^{n}\right)$ such that $\varphi$ vanishes on a neighborhood of $\Omega^{c}$ and $\varphi(x)=1$ outside some compact set. The above argument proves that $u_{\varepsilon} \rightarrow u$ in $L_{\text {loc }}^{2}(\Omega)$. We complete the proof by showing that $\varphi u_{\varepsilon} \rightarrow \varphi u$ in $L^{2}(\Omega)$. It suffices to show that $\left\{\varphi u_{\varepsilon}\right\}$ is a Cauchy sequence in $L^{2}(\Omega)$ since we already know 
that $\varphi u_{\varepsilon} \rightarrow \varphi u$. Subtract equation (2.7) for one value $\varepsilon_{1}$ of $\varepsilon$ from the same equation for a different value $\varepsilon_{2}$, multiply by $\varphi^{2}\left(u_{\varepsilon_{1}}-u_{\varepsilon_{2}}\right)$ and integrate by parts to find

$$
\left(\delta-\delta_{0}\right)\left\|\varphi u_{\varepsilon_{1}}-\varphi u_{\varepsilon_{2}}\right\|_{L^{2}(\Omega)}^{2} \leqslant c\left(\varepsilon_{1}\left\|u_{\varepsilon_{1}}\right\|_{H_{\tan }^{1}(\Omega)}^{2}+\varepsilon_{2}\left\|u_{\varepsilon_{2}}\right\|_{H_{\tan }^{1}(\Omega)}^{2}\right) .
$$

Only the tangential norms occur since $\varphi \equiv 0$ near $\partial \Omega$. By Theorem 5 the right-hand side converges to zero as $\varepsilon_{1}$ and $\varepsilon_{2}$ converge to zero and the proof is complete.

Proof of Theorem 6. Part (1). By Theorem 5 we see that if $\delta>\delta_{1},\left\|u_{\varepsilon}\right\|_{H_{\tan }^{1}(\Omega)}<$ $c\|f\|_{H_{\tan }^{1}(\Omega)}$. To prove the theorem it suffices to show that the localizations, $u_{\varepsilon}^{l}=\left(\chi_{l} u_{\varepsilon}\right) \circ \tau_{l}^{-1}, l=1,2, \ldots$, as in (4.1), (4.2), satisfy

$$
\left\|\left(I-p_{0}\right) u_{\varepsilon}^{l}\right\|_{H^{1,2-\eta}\left(\mathbf{R}_{-}: L^{2}\left(\mathbf{R}^{n-1}\right)\right)} \leqslant c\|f\|_{H_{\text {tan }}^{1}}
$$

where $p_{0}=P_{0} \circ \tau^{-1}$.

Theorem 5 shows that $u_{\varepsilon}^{l}$ is bounded in $L^{2}\left(\mathbf{R}_{-} ; H^{1}\right)$ and $\varepsilon^{1 / 2} u_{\varepsilon}^{l}$ is bounded in $\stackrel{\circ}{H}_{1}\left(\mathbf{R}_{-} ; H^{1}\right)$ where for ease of reading we abbreviate $H^{1}\left(\mathbf{R}^{n-1}\right)$ to $H^{1}$ when confusion is unlikely. Spaces $L^{2}\left(\mathbf{R}^{n-1}\right)$ and $H^{-1}\left(\mathbf{R}^{n-1}\right)$ will be treated similarly. We may write the differential equation satisfied by $u_{\varepsilon}^{l}$ with all the tangential derivative terms on the right-hand side to obtain

$$
-\varepsilon D_{1} e_{11} D_{1} u_{\varepsilon}^{l}+a_{1} D_{1} u_{\varepsilon}^{l}+\delta u_{\varepsilon}^{l}=g_{\varepsilon}
$$

with $\left\|g_{\varepsilon}\right\|_{L^{2}\left(\mathbf{R}_{\sim}: L^{2}\right)} \leqslant c\|f\|_{H_{\tan }^{1}(\Omega)}$. Let $w_{\varepsilon}=e_{11}^{1 / 2} u_{\varepsilon}^{l}$ (the $l$ dependence of $w_{\varepsilon}$ is suppressed); then if $\tilde{a}=e_{11}^{-1 / 2} a_{1} e_{11}^{-1 / 2}$, we have

$$
-\varepsilon D_{1}^{2} w_{\varepsilon}+\tilde{a} D_{1} w_{\varepsilon}+\delta e_{11}^{-1} w_{\varepsilon}=\tilde{g}_{\varepsilon} \text { in } \Re_{-}
$$

where $\tilde{g}_{\varepsilon}$ is bounded in the same way as $g_{\varepsilon}$. Extend $e_{11}$ and $\tilde{a}$ from $\mathscr{B}_{-}$to $\mathbf{R} \times \mathbf{R}^{n-1}$ to be $C^{2}$ functions independent of $x$ for $|x|$ large and so that $e_{11}$ is positive definite for all $x$. Extend $w_{\varepsilon}$ and $\tilde{g}_{\varepsilon}$ to all of $\mathbf{R} \times \mathbf{R}^{n-1}$ by setting them equal to zero in $\mathbf{R}_{+} \times \mathbf{R}^{n-1}$. Denoting the resulting functions by the same symbols $w_{\varepsilon}, \tilde{g}_{\varepsilon}$, we have

$$
-\varepsilon D_{1}^{2} w_{\varepsilon}+\tilde{a} D_{1} w_{\varepsilon}+\delta e_{11}^{-1} w_{\varepsilon}=\tilde{g}_{\varepsilon}+\delta\left(x_{1}\right) \otimes \varepsilon D_{1} w_{\varepsilon}(0) \quad \text { in } \mathbf{R} \times \mathbf{R}^{n-1}
$$

where

$$
\varepsilon D_{1} w_{\varepsilon}(0)=\lim _{x_{1}>0} D_{1} w_{\varepsilon}\left(x_{1}\right)
$$

exists in $H^{1 / 2}\left(\mathbf{R}^{n-1}\right)$ since $w_{\varepsilon} \in H^{2}\left(\mathbf{R}_{-} \times \mathbf{R}^{n-1}\right)$. The next lemma studies the regularity of solutions to this equation. A crucial idea is to use the multiplier $\tilde{a} D_{1} w_{\varepsilon}$.

LEMMA 5. There are positive constants $c$ and $\delta_{5}$ so that for any $s \in[-1,1]$, $r \in[-1,1], \delta>\delta_{5}, 0<\varepsilon<1$, and $g \in H^{s}\left(\mathbf{R}: H^{r}\left(\mathbf{R}^{n-1}\right)\right)$, there is a unique $v \in$ $H^{s+2}\left(\mathbf{R}: H^{r}\left(\mathbf{R}^{n-1}\right)\right)$ such that

$$
-\varepsilon D_{1}^{2} v+\tilde{a} D_{1} v+\delta e_{11}^{-1} v=g
$$

and, in addition,

$$
\begin{gathered}
\|v\|_{H^{s}\left(\mathbf{R}: H^{r}\left(\mathbf{R}^{n-1}\right)\right)} \leqslant c \delta^{-1}\|g\|_{H^{s}\left(\mathbf{R}: H^{r}\left(\mathbf{R}^{n-1}\right)\right)}, \\
\|\tilde{a} v\|_{H^{s+1}\left(\mathbf{R}: H^{r}\left(\mathbf{R}^{n-1}\right)\right)} \leqslant c\|g\|_{H^{s}\left(\mathbf{R}: H^{r}\left(\mathbf{R}^{n-1}\right)\right)}
\end{gathered}
$$


Proof OF LEMMA. Except for estimate (5.8) this result is standard. The estimate (5.7) for $s=0$ and $r=0$ is proved with the multiplier $v$ and for $r=1$ with multiplier $\sum_{j=2} D_{j}^{2} v$. By interpolation the result follows for $s=0$ and $0 \leqslant r \leqslant 1$. For $s=1$ the multipliers $D_{1}^{2} v$ and $\sum_{j=2} D_{1}^{2} D_{j}^{2} v$ yield estimate (5.7) for $r=0$ and 1, respectively. By interpolation (5.7) follows for $0 \leqslant s \leqslant 1,0 \leqslant r \leqslant 1$. The values $-1 \leqslant s \leqslant 0,-1 \leqslant r \leqslant 0$ are obtained by duality.

The critical additional step in proving (5.8) is to use the multiplier $\tilde{a} D_{1} v$ to obtain

$$
\begin{aligned}
& -\varepsilon \operatorname{Re} \int_{\mathbf{R} \times \mathbf{R}^{n}}\left\langle D_{1}^{2} v, \tilde{a} D_{1} v\right\rangle+\int_{\mathbf{R} \times \mathbf{R}^{n}}\left\|\tilde{a} D_{1} v\right\|^{2} \\
& \quad \leqslant c\left(\delta\|v\|_{L^{2}\left(\mathbf{R} \times \mathbf{R}^{n-1}\right)}+\|g\|_{L^{2}\left(\mathbf{R} \times \mathbf{R}^{n-1}\right)}\right)\left(\left\|\tilde{a} D_{1} v\right\|_{L^{2}\left(\mathbf{R} \times \mathbf{R}^{n-1}\right)}\right)
\end{aligned}
$$

The right-hand side is dominated by $c\|g\|_{L^{2}\left(\mathbf{R} \times \mathbf{R}^{n-1}\right)}\left\|\tilde{a} D_{1} v\right\|_{L^{2}\left(\mathbf{R} \times \mathbf{R}^{n}\right)}$ by virtue of (5.7) and

$$
-\varepsilon \operatorname{Re} \int_{\mathbf{R}_{-} \times \mathbf{R}^{n-1}}\left\langle D_{1}^{2} v, \tilde{a} D_{1} v\right\rangle=-\frac{\varepsilon}{2} \int_{\mathbf{R}_{-} \times \mathbf{R}^{n-1}}\left\langle D_{1} v,\left(D_{1} \tilde{a}\right) D_{1} v\right\rangle
$$

is bounded by a constant times $\|g\|_{L^{2}\left(\mathbf{R} \times \mathbf{R}^{n-1}\right)}^{2}$ by the standard energy estimate (multiplier $=v$ ). This proves (5.8) for $s=r=0$. For $s=0, r=1$ the multiplier is $\sum_{j=2}^{n} \tilde{a} D_{1} D_{j}^{2} v$ and for $s=1, r=1$, the multiplier $\sum_{j=2}^{n} \tilde{a} D_{1}^{3} D_{j}^{2} v$ is used. The general case follows by interpolation and duality.

We can estimate the trace $D_{1} w_{\varepsilon}(0)$ using

$$
\left\|\varepsilon D_{1} w_{\varepsilon}(0)\right\|_{H^{1 / 2}\left(\mathbf{R}^{n-1}\right)} \leqslant c\left\|\varepsilon D_{1} w_{\varepsilon}\right\|_{L^{2}\left(\mathbf{R}_{-}: H^{1}\right)}\left\|\varepsilon D_{1}^{2} w_{\varepsilon}\right\|_{L^{2}\left(\mathbf{R}_{-}: L^{2}\right)} .
$$

The basic tangential regularity estimate in part (1) of Theorem 5 implies that

$$
\varepsilon^{1 / 2}\left\|D_{1} w_{\varepsilon}\right\|_{L^{2}\left(\mathbf{R}_{-}: H^{1}\right)} \leqslant c\|f\|_{H_{\tan (\Omega)}^{1}}
$$

and the function $\varepsilon D_{1}^{2} w_{\varepsilon}$ is estimated using the differential equation (5.4):

$$
\left\|\varepsilon D_{1}^{2} w_{\varepsilon}\right\|_{L^{2}\left(\mathbf{R}_{-}: L^{2}\right)}=\left\|\tilde{a} D_{1} w_{\varepsilon}+\delta e_{11}^{-1} w_{\varepsilon}-g_{\varepsilon}\right\|_{L^{2}\left(\mathbf{R}_{-}: L^{2}\right)} .
$$

The basic energy estimate (3.1) for $u_{\varepsilon}$ implies that the norms of $\delta w$ and $\varepsilon^{1 / 2} D_{1} w$ are bounded by $c\|f\|_{H_{\tan }^{1}}$. Thus,

$$
\left\|\varepsilon D_{1}^{2} w_{\varepsilon}\right\|_{L^{2}\left(\mathbf{R}_{-}: L^{2}\right)} \leqslant c \varepsilon^{-1 / 2}\|f\|_{H_{\tan }^{1}(\Omega)} .
$$

In total, $\left\|\varepsilon D_{1} w_{\varepsilon}(0)\right\|_{H^{1 / 2}\left(\mathbf{R}^{n-1}\right)} \leqslant c\|f\|_{H_{\tan }^{1}(\Omega)}$. Then, for any $0<\eta \leqslant \frac{1}{2}$,

$$
\left\|\delta\left(x_{1}\right) \otimes \varepsilon D_{1} w_{\varepsilon}(0)\right\|_{H^{-1 / 2-\eta}\left(\mathbf{R}: H^{1 / 2}\left(\mathbf{R}^{n-1}\right)\right)} \leqslant c\|f\|_{H_{\mathrm{tan}}^{1}(\Omega)} .
$$

Thus, the right-hand side of (5.5) is bounded in $H^{-1 / 2-\eta}\left(\mathbf{R}: L^{2}\left(\mathbf{R}^{n-1}\right)\right)$ and Lemma 5 yields the important estimate

$$
\left\|\tilde{a} w_{\varepsilon}\right\|_{H^{1 / 2-\eta}\left(\mathbf{R}: L^{2}\left(\mathbf{R}^{n-1}\right)\right)} \leqslant c\|f\|_{H_{\mathrm{tan}}^{\mathrm{1}(\Omega)}},
$$

which implies (5.3) and therefore completes the proof of part (1).

REMARK 1. If $f \in H_{\mathrm{tan}}^{2}(\Omega)$, reasoning as above shows that $\tilde{g}_{\varepsilon}$ in (5.4) is uniformly bounded in $L^{2}\left(\mathbf{R}_{-}: H^{1}\left(\mathbf{R}^{n}\right)\right)$ and $\left\|\varepsilon D_{1} w(0)\right\|_{H^{3 / 2}\left(\mathbf{R}^{n-1}\right)} \leqslant c\|f\|_{H_{\text {tan }}^{2}(\Omega)}$. Thus we have a bound on $\tilde{g}_{\varepsilon}+\delta \otimes \varepsilon D_{1} w(0)$ in $H^{-1 / 2-\eta}\left(\mathbf{R}: H^{1}\right)$ and the corresponding estimate

$$
\left\|\left(I-p_{0}\right) u_{\varepsilon}^{l}\right\|_{H^{1 / 2-\eta}\left(\mathbf{R}_{-}: H^{\prime}\left(\mathbf{R}^{n-1}\right)\right)} \leqslant c\|f\|_{H_{\text {tan }}^{2}(\Omega)}
$$


follows if $a_{1}$ is three times differentiable and $e_{11}$ is twice differentiable. This estimate is needed in the proof of part (2).

Proof of Theorem 6. Part (2). In the notation of the proof of part (1) we need to prove

$$
\left\|p_{0} w_{\varepsilon}\right\|_{H^{1 / 2-\eta}\left(\mathbf{R}_{-}: L^{2}\right)} \leqslant c\left(\|f\|_{H_{\tan (\Omega)}^{2}(\Omega)}+\left\|P_{0} f\right\|_{H^{1}(\Omega)}\right) .
$$

The basic idea is that multiplying equation (5.4) by $P_{0}$ eliminates the term $\tilde{a} D_{1} w_{\varepsilon}$ and $P_{0} w_{\varepsilon}$ can be estimated from the resulting equations.

First, observe that taking a different (finer) open cover $O_{l}$ if necessary and making a smooth orthogonal change of basis in $\mathbf{C}^{k}$ we may suppose that for $z \in \mathbf{C}^{k}$ and $x \in \operatorname{supp} w_{\varepsilon}$ that $p_{0} z=\pi_{0} z=\left(z_{1}, z_{2}, \ldots, z_{l}, 0,0, \ldots, 0\right)$.

Next, we make a closer analysis of the equation satisfied by $w_{\varepsilon}$. In (5.4) all tangential derivatives were absorbed in $\tilde{g}_{\varepsilon}$; here we will separate the term $\sum_{1}^{n} \tilde{a}_{k} D_{k} w_{\varepsilon}$ so

$$
-\varepsilon D_{1}^{2} w_{\varepsilon}+\sum_{1}^{n} \tilde{a}_{k} D_{k} w_{\varepsilon}+\delta \tilde{e}_{11}^{-1} w_{\varepsilon}+b w_{\varepsilon}=h_{\varepsilon}+k_{\varepsilon}
$$

where $b$ is bounded independently of $\delta$, and $k_{\varepsilon}$ denotes the terms other than $-\varepsilon D_{1}^{2} w$ which contain a factor of $\varepsilon$. For these we have the trivial estimate

$$
\left\|k_{\varepsilon}\right\|_{L^{2}\left(\mathbf{R}_{-}: H^{1}\right)} \leqslant c \varepsilon\|\nabla u\|_{H_{\text {tan }}^{2}} .
$$

The tangential regularity yields

$$
\left\|k_{\varepsilon}\right\|_{L^{2}\left(\mathbf{R}_{-}: H^{\prime}\right)}^{2} \leqslant c \varepsilon \delta^{-1}\|f\|_{H_{\text {tan }}^{2}(\Omega)}^{2}
$$

and

$$
\left\|p_{0} h_{\varepsilon}\right\|_{H^{\prime}\left(\mathbf{R}_{-} \times \mathbf{R}^{n-1}\right)} \leqslant c\left\|p_{0} f\right\|_{H^{\prime}(\Omega)} .
$$

The equation for $w_{\varepsilon}$ is broken into the components in range $p_{0}$ and the rest. Toward this end, let $p_{1}=I-p_{0}, w_{\varepsilon}^{1}=p_{1} w_{\varepsilon}, w_{\varepsilon}^{0}=p_{0} w_{\varepsilon}$. The remark following the proof of part (1) shows that

$$
\left\|w_{\varepsilon}^{1}\right\|_{H^{1 / 2-\eta}\left(\mathbf{R}_{-}: H^{1}\left(\mathbf{R}^{n-1}\right)\right)} \leqslant c\|f\|_{H_{\tan }^{2}(\Omega)},
$$

so for $j \geqslant 2$,

$$
\left\|a_{j} D_{j} w_{\varepsilon}^{1}\right\|_{H^{1 / 2-\eta}\left(\mathbf{R}_{-}: L^{2}\left(\mathbf{R}^{n-1}\right)\right)} \leqslant c\|f\|_{H_{\tan }^{2}(\Omega)} .
$$

The $a_{j} D_{j} w_{\varepsilon}^{0}$ part of $a_{j} D_{j} w$ is written as (note $D_{j} p_{0}=0$ )

$$
a_{j} D_{j} w_{\varepsilon}^{0}=a_{j} D_{j} p_{0}^{2} w_{\varepsilon}^{0}=a_{j} p_{0} D_{j} w_{\varepsilon}^{0} .
$$

Thus, multiplying (5.11) by $p_{0}$ yields

$$
\begin{aligned}
-\varepsilon D_{1}^{2} w_{\varepsilon}^{0} & +\sum_{j=2}^{n} p_{0} a_{j} p_{0} D_{j} w_{\varepsilon}^{0}+\delta p_{0} e_{11}^{-1} p_{0} w_{\varepsilon}^{0}+p_{0} b w_{\varepsilon}^{0} \\
& =p_{0}\left(k_{\varepsilon}+h_{\varepsilon}-\delta e_{11}^{-1} w_{\varepsilon}^{1}-b w_{\varepsilon}^{1}-\sum_{j=2}^{n} a_{j} D_{j} w_{\varepsilon}^{1}\right) .
\end{aligned}
$$


Since $p_{0}$ is just projection on the first $l$ variables, we may let $v=\left(\left(w_{\varepsilon}^{0}\right)_{1}, \ldots,\left(w_{\varepsilon}^{0}\right)_{l}\right)$ to obtain $v \in \stackrel{\circ}{H}^{1}\left(\mathbf{R}_{-} \times \mathbf{R}^{n}\right) \cap H^{2}\left(\mathbf{R}_{-} \times \mathbf{R}^{n}\right)$ and

$$
-\varepsilon D_{1}^{2} v+\sum_{j=2}^{n} r_{j}(x) D_{j} v+\delta q(x) v+\tilde{b}(x) v=g
$$

where $g$ is the first $l$ component of the right-hand side of (5.13), $r_{j}, \tilde{b}$, and $q$ are $C^{2}\left(\mathbf{R}^{n}: \operatorname{Hom}\left(\mathbf{C}^{l}\right)\right)$ with $r_{j}$ and $q$ hermitian, $q$ positive definite and all independent of $x$ for $|x|$ large. We use two estimates for the solutions to (5.14), both valid uniformly for $0<\varepsilon$ and $\delta$ large,

$$
\begin{gathered}
\delta\|v\|_{H^{1 / 2-\eta}\left(\mathbf{R}: L^{2}\right)} \leqslant c\|g\|_{H^{1 / 2-\eta}\left(\mathbf{R}: L^{2}\right)}, \\
\varepsilon \delta\|v\|_{H^{1}\left(\mathbf{R}_{-}: L^{2}\right)}^{2} \leqslant c\|g\|_{L^{2}\left(\mathbf{R}_{-} \times \mathbf{R}^{n-1}\right)}^{2} .
\end{gathered}
$$

Postponing the derivation of these estimates, we complete the proof. Write $g=g_{1}+g_{2}$ where $g_{1}$ is the first $l$ components of $h_{\varepsilon}-\delta e_{11}^{-1} w_{\varepsilon}^{1}-b w_{\varepsilon}^{1}-\sum_{j \geqslant 2}^{n} a_{j} D_{j} w_{\varepsilon}^{1}$ and $g_{2}$ the same components of $k_{\varepsilon}$ and let $v_{1}$ and $v_{2}$ be the corresponding solutions of (5.14) with $v_{i} \in \stackrel{\circ}{H}^{1}\left(\mathbf{R}_{-}: L^{2}\right)$; then $v=v_{1}+v_{2}$ and (5.12), together with (5.16) show that

$$
\delta\left\|v_{2}\right\|_{H^{1}\left(\mathbf{R}_{-}: L^{2}\right)} \leqslant c\|f\|_{H_{\tan }^{2}(\Omega)} .
$$

To estimate $v_{1}$ we use (5.15). This requires an estimate for $g_{1}$ in $H^{1 / 2-\eta}\left(\mathbf{R}_{-}: L^{2}\right)$. Inequality $(5.12)_{2}$ bounds $p_{0} h_{\varepsilon}$ in this space. On the other hand (5.9) shows that

$$
\left\|\delta e_{11}^{-1} w_{\varepsilon}^{1}+b w_{\varepsilon}^{1}+\sum_{j \geqslant 2} a_{j} D_{j} w_{\varepsilon}^{1}\right\|_{H^{1 / 2-\eta}\left(\mathbf{R}_{-}: L^{2}\right)} \leqslant c\left(\delta\|f\|_{H_{\mathrm{tan}}^{1}}+\|f\|_{H_{\mathrm{tan}}^{2}}\right) .
$$

This together with the estimate for $p_{0} h_{\varepsilon}$ yields

$$
\left\|g_{1}\right\|_{H^{1 / 2}\left(\mathbf{R}_{-}: L^{2}\right)} \leqslant c \delta\left(\left\|p_{0} f\right\|_{H^{1}(\Omega)}+\|f\|_{H_{\tan }^{2}(\Omega)}\right) .
$$

Estimate (5.15) yields

$$
\left\|v_{1}\right\|_{H^{1 / 2-\eta}\left(\mathbf{R}_{-}: L^{2}\right)} \leqslant c\left(\left\|p_{0} f\right\|_{H^{1}(\Omega)}+\|f\|_{H_{\tan }^{2}(\Omega)}\right) .
$$

Adding the estimates for $v_{1}$ and $v_{2}$ yields, for large $\delta$,

$$
\|v\|_{H^{1 / 2-\eta}\left(\mathbf{R}_{-}: L^{2}\right)} \leqslant c\left(\left\|p_{0} f\right\|_{H^{1}(\Omega)}+\|f\|_{H_{\mathrm{tan}}^{2}(\Omega)}\right) .
$$

Since

$$
\left\|p_{0} w_{\varepsilon}\right\|_{H^{1 / 2-\eta}\left(\mathbf{R}_{-}: L^{2}\right)}=\|v\|_{H^{1 / 2-\eta}\left(\mathbf{R}_{-}: L^{2}\right)},
$$

this proves (5.10). Thus, to complete the proof of Theorem 6 , it suffices to prove estimates (5.15) and (5.16) for the solutions $v \in \stackrel{H}{ }^{1}\left(\mathbf{R}_{-} \times \mathbf{R}^{n-1}\right)$ of (5.14).

The proof of (5.15) proceeds in several steps, each one an application of the energy method with a different multiplier. First, one shows that, for $\delta$ large,

$$
\delta\|v\|_{L^{2}\left(\mathbf{R}_{-}: L^{2}\right)} \leqslant c\|g\|_{L^{2}\left(\mathbf{R}: L^{2}\right)} .
$$


This is proved by multiplying (5.14) by $v$ and integrating over $\mathbf{R}_{-} \times \mathbf{R}^{n-1}$ to obtain

$$
\varepsilon \int\left|D_{1} v\right|^{2}+c(\delta-c) \int|v|^{2} \leqslant\left(\int|v|^{2}\right)^{1 / 2}\left(\int|g|^{2}\right)^{1 / 2}
$$

which also proves (5.16). Next, one shows that

$$
\delta\|v\|_{H^{1}\left(\mathbf{R}_{-}: L^{2}\right)} \leqslant c\|g\|_{H^{1}\left(\mathbf{R}_{-}: L^{2}\right)} .
$$

This is proved using the multiplier $D_{1}^{2} v$. Interpolating between (5.17) and (5.18) yields

$$
\delta\|v\|_{H_{\theta}} \leqslant c\|g\|_{H_{\theta}}, \quad 0 \leqslant \theta \leqslant 1,
$$

where $H_{\theta}$ is the interpolation space $\left[L^{2}\left(\mathbf{R}_{-}: L^{2}\right), \stackrel{\circ}{H}^{1}\left(\mathbf{R}_{-}: L^{2}\right)\right]_{\theta}$. For $0 \leqslant \eta<\frac{1}{2}$ and $\theta=\frac{1}{2}-\eta, H_{\theta}=H^{1 / 2-\eta}\left(\mathbf{R}_{-}: L^{2}\right)$ so (5.19) for this value of $\theta$ is exactly (5.15) and the proof is complete.

\section{BIBLIOGRAPHY}

1. C. Bardos, D. Brézis and H. Brézis, Perturbations singulières et prolongements maximaux d'opérateurs positifs, Arch. Rational Mech. Anal. 53 (1973), 69-100.

2. K. O. Friedrichs, Symmetric hyperbolic linear differential equations, Comm. Pure Appl. Math. 7 (1954), 345-392.

3. . Symmetric positive linear differential equations, Comm. Pure Appl. Math. 11 (1958), $333-418$.

4. Well-posed problems of mathematical physics, mimeographed lecture notes, New York Univ.

5. L. Hörmander, Linear partial differential operators, 2nd rev. printing, Springer-Verlag, Berlin, 1964.

6. T. Kato, Perturbation theory for linear operators, Springer-Verlag, New York, 1966.

7. Singular perturbation and semi group theory, Turbulence and the Navier Stokes Equation, Lecture Notes in Math., vol. 565, Springer-Verlag, Berlin, 1976, pp. 104-112.

8. P. D. Lax and R. S. Phillips, Local boundary conditions for dissipative symmetric linear differential operators, Comm. Pure Appl. Math. 13 (1960), 427-456.

9. J. L. Lions, Perturbations singulière dans les problèmes aux limites et en contrôl optimal, Lecture Notes in Math., vol. 323, Springer-Verlag, Berlin, 1973.

10. J. Rauch and M. Taylor, Potential and scattering theory on wildly perturbed domains, J. Funct. Anal. 18 (1975), 27-59.

11. L. Sarason, On weak and strong solutions of boundary value problems, Comm. Pure Appl. Math. 15 (1962), 237-288.

12. , Differentiable solutions of symmetrizable and singular symmetric first order systems, Arch. Rational Mech. Anal. 26 (1967), 357-384.

13. D. Tartakoff, Regularity of solutions to boundary value problems for first order systems, Indiana Univ. Math. J. 21 (1972), 1113-1129.

14. M. I. Visik and L. A. Lyusternik, Regular degeneration and boundary layers for linear differential equations with small parameter, Uspehi Mat. Nauk (N.S.) 12 (1957), 3-122; Amer. Math. Soc. Transl. (2) 20 (1962), 239-364.

15. The asymptotic behavior of solutions of linear differential equations with large or quickly changing coefficients and boundary condition, Russian Math. Surveys 4 (1960), 23-92.

16. C. Wilcox, Wave operators and asymptotic solutions of wave propagation problems of classical physics, Arch. Rational Mech. Anal. 22 (1966), 37-78.

17. J. Rauch, Boundary value problems as limits of problems in all space, Séminaire Goulaouic-Schwartz, École Polytechnique, Exposé III, 1978.

Departement de Mathematiques, Universite Paris-Nord, Paris, France

Department of Mathematics, University of Michigan, Ann Arbor, Michigan 48104 